\title{
Video Game-Based Therapy on Balance and Gait of Patients with Stroke: A Systematic Review
}

\author{
Silvia Pintado-Izquierdo ${ }^{1}$, Roberto Cano-de-la-Cuerda ${ }^{2, *}$ (i) and Rosa María Ortiz-Gutiérrez ${ }^{3}$ \\ 1 Escuela Internacional de Doctorado, Universidad Rey Juan Carlos, Alcorcón, 28922 Madrid, Spain; \\ s.pintadoi@alumnos.urjc.es \\ 2 Department of Physiotherapy, Occupational Therapy, Rehabilitation and Physical Medicine, \\ Health Sciences Faculty, Rey Juan Carlos University, Avda. Atenas s/n. 28922, Alcorcón, 28922 Madrid, Spain \\ 3 Department of Physiotherapy, Health Sciences Faculty San Rafael-Nebrija, FSJD. 28922 Madrid, Spain; \\ rortizg@nebrija.es \\ * Correspondence: roberto.cano@urjc.es; Tel.: +34-91-488-86-74
}

Received: 27 July 2020; Accepted: 12 September 2020; Published: 15 September 2020

\begin{abstract}
Background: Stroke patients with motor, sensory and cognitive diseases can take profits from information and communication technologies-in particular, from the latest commercial video consoles, which are based on motion capture. These technologies are positioning themselves as complementary therapeutic tools for treating gait and balance disorders. In this paper, a systematic review of the effect of video game-based therapy on balance and gait in stroke patients is shown and compared with other types of treatments. Methods: A systematic review of prospective controlled clinical trials published in the main biomedical databases in English and Spanish between 2005 and 2020 was performed. The systematic review presented in this paper has been done following the Cochrane Manual recommendations and the PRISMA Declaration by two independent reviewers. Data about participants, intervention, outcome measurements and outcome measurement results were extracted. The quality of evidence of each study was assessed using Cochrane's standard quality assessment format, which includes a description of the risk of bias. Additionally, the Physiotherapy Evidence Database (PEDro) scale was used to assess the methodological quality of each paper. Results: A total of 18 papers, including 479 patients, were included in this systematic review, in which the use of video consoles (in combination with conventional rehabilitation or exclusively) was compared with conventional rehabilitation to treat balance or gait in post-stroke patients. In all studies, a tendency to improve balance was found in both intervention groups, finding, in 10 of 17 studies that analysed it, a better capacity in the experimental group that included video consoles compared to the conventional rehabilitation control group. Regarding gait, in six of seven studies that analysed it, improvements were found in both intervention groups, and these improvements were greater in the experimental group than compared to the control group in three of them. Conclusions: Commercial video game systems, in combination with conventional rehabilitation, have shown positive results on balance and gait in post-stroke patients. There were variations between the trials in terms of the video consoles used and the duration, frequency and number of sessions with commercial video games. Future studies should compare the effects of commercial video game treatments on balance and gait in stroke patients with a nonintervention group to know their real efficacy.
\end{abstract}

Keywords: commercial gaming devices; balance; gait; stroke; virtual reality; video consoles; video games

\section{Introduction}

Stroke is defined as a "focal neurological condition (or sometimes general) which occurs suddenly, lasting for more than $24 \mathrm{~h}$ (or the cause of death) and supposedly of vascular origin" [1]. According to 
the World Health Organization (WHO), the average incidence of stroke is around 200 new cases per 100,000 inhabitants/year [2,3]. Stroke induces sensory, motor, cognitive and psychological deficits, which directly affect the individual's functionality and quality of life [1]. In this context, between $50 \%$ and $70 \%$ of stroke patients present with balance and gait disorders and frequent falls $[4,5]$. Therefore, balance and gait disturbances in stroke patients are a key objective for the rehabilitation team.

Continued rehabilitation during the first year after stroke reduces the risk of functional impairment and improves independence in activities of daily living. In the chronic phase, continuity in rehabilitation programmes is also necessary due to the appearance of associated functional deterioration [2]. In this context, virtual reality (VR) has become extremely relevant in the rehabilitation of cognitive and motor disorders in these patients. VR has been defined as the "use of interactive simulations created with computer hardware and software to present users with opportunities to engage in environments that appear and feel similar to real-world objects and events" [6]. VR possesses a highly motivating multidimensional virtual environment that the user can interact with, which provides sensory feedback, thus allowing the individual to perform in activities or tasks comparable to real situations. The intensity and difficulty degree can be graded and provides the user with information about the reached objectives in real time [7-10]. There are different types of VR, including: immersive, in which the patient is fully integrated in the virtual environment and interacts with it through some type of hardware, such as data gloves or VR glasses, semi-immersive, in which the patient is visualised within the virtual environment through a screen and interacts with it through their movements or with some remote controller but without losing contact with the real world, and nonimmersive, in which the patient interacts with the virtual environment through a computer-generated character represented on a screen with a peripheral device controller [8,11-13]. Immersive VR systems have shown good results in the treatment of motor deficits, among which are balance and gait alterations, in stroke patients. However, there is no suitable protocol, and these systems are costly and complex to use and maintain, unlike semi-immersive low-cost video game systems [14-16].

Video games have experienced a technological growth phenomenon which, apart from constituting a leisure activity, are considered an important tool for learning and motor programme training in individuals with motor, cognitive and sensory deficits of a neurological origin. Within the available systems, commercial video games based on motion capture are an alternative way of providing semi-immersive VR for rehabilitation approaches, such as the possibility of practising functional activities in a controlled environment, and an increased adherence to the therapy, as it is highly motivating for the patient. Their low cost, accessibility and user-friendliness allows their use as a rehabilitation tool [17-19].

Many systematic reviews (SRs) and meta-analyses have been published in recent years analysing the effects of VR on balance and gait in stroke patients. Studies comparing the impacts of VR often include heterogeneous systems or technologies that are customised or noncustomised for stroke treatment, addressing a broad range of disabilities. However, effectiveness can only be investigated if similar systems that rehabilitate the same impairment are contrasted. On the other hand, a clear understanding of the benefits that should make VR interventions effective in promoting recovery is missing. To our knowledge, few studies have specifically analysed the effects of commercial video games in stroke patients [14-16,20-22]. In addition, the appearance on the market of new generation video consoles make it necessary to know the main results provided by these devices used to date in order to propose rehabilitation strategies in patients with stroke using new video consoles.

The aim of this paper is to carry out a SR on the effects of video game-based therapy on balance and gait in patients who have suffered a stroke compared to other types of treatment and to analyse the differences between the commercial devices and video games used. 


\section{Materials and Methods}

\subsection{Design}

A paired systematic review (SR) was performed, following the Cochrane Manual recommendations for SR, as well as the Preferred Reporting Items for Systematic Reviews and Meta-Analyses (PRISMA) Declaration $[23,24]$.

\subsection{Search Strategy}

Two independent reviewers carried out bibliographic searches in the following electronic databases: CINAHL Medical Science, Medline through EBSCO, PubMed, Physiotherapy Evidence Database (PEDro) and Scopus, limiting the search to papers from January 2005 to June 2020 in English and Spanish.

Inverse searches were also carried out based on papers found in the databases. The reviewers used the COVIDENCE ${ }^{\circledR}$ application (V.1.0. Covidence, Melbourne, VIC, Australia) to sift through the papers, and a third reviewer worked on resolving discrepancies between the papers belonging to and being included in this SR.

The search strategy is shown in Table 1.

Table 1. Search strategy.

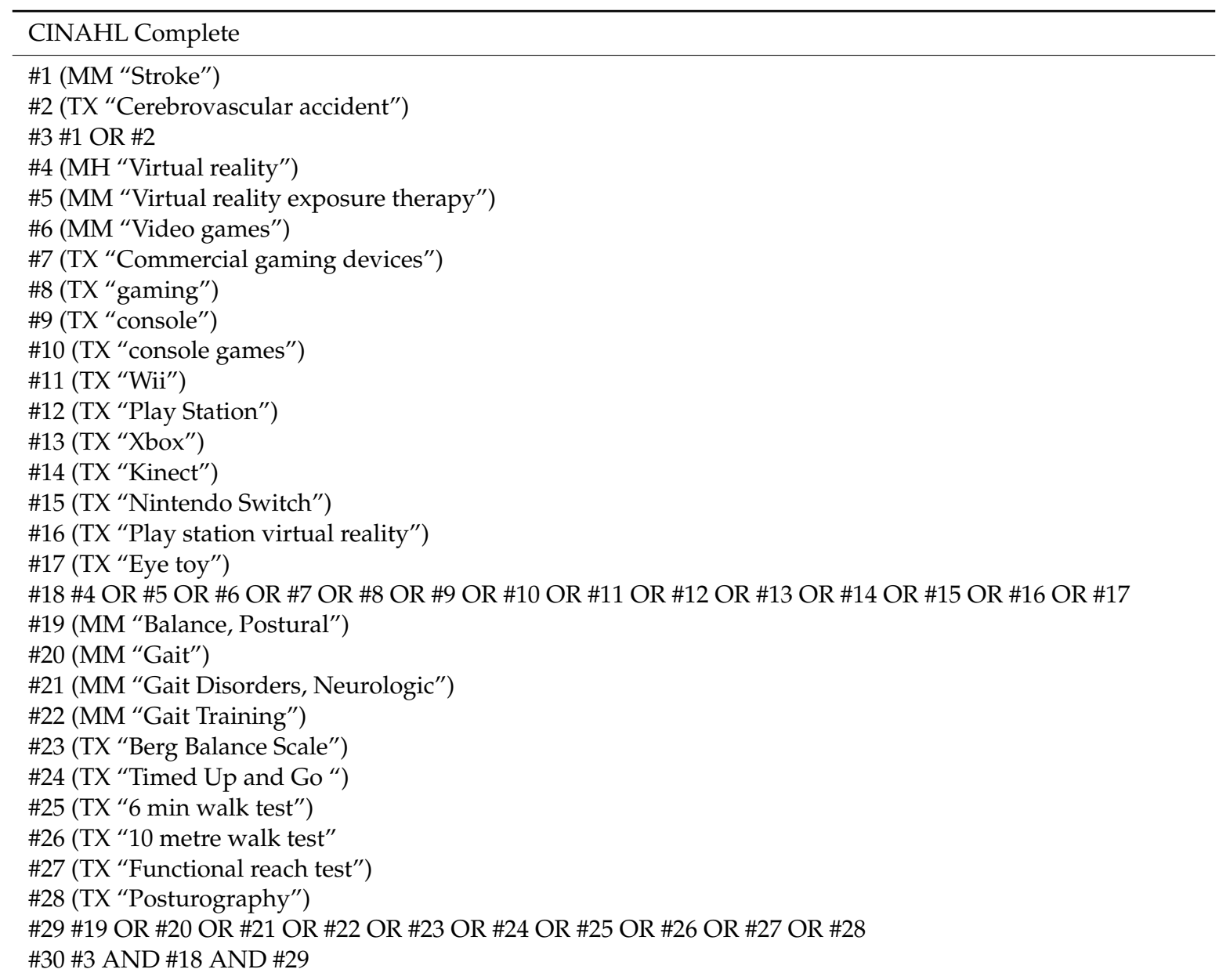


Table 1. Cont.
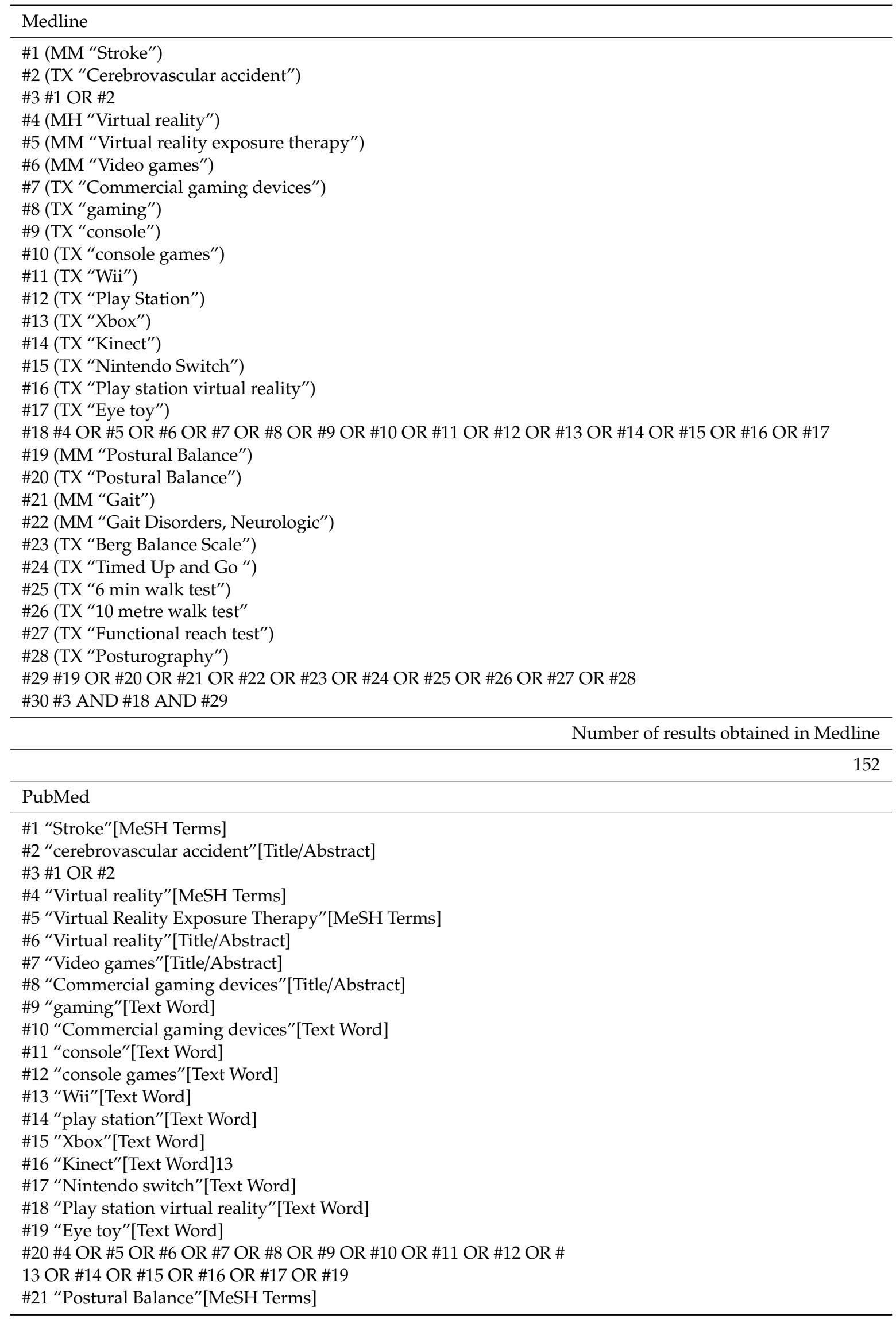
Table 1. Cont.

\#22 “Gait”[MeSH Terms]

\#23 “Gait Disorders, Neurologic"[MeSH Terms]

\#24 "Gait training"[Text Word]

\#25 “Berg Balance Scale"[Text Word]

\#26 "Timed Up and Go"[Text Word]

\#27 "6 min walk test" [Text Word]

\#28 "10 metre walk test"[Text Word]

\#29 "Posturography"[Text Word]

\#30 “Functional reach test"[Text Word]

\#31 "Balance" [Title/Abstract]

\#32 \#21 OR \#22 OR \#23 OR \#24 OR \#25 OR \#26 OR \#27 OR \#28 OR \#29 OR \#30 OR \#31

\#33 \#3 AND \#20 AND \#32

\begin{tabular}{lr}
\hline & Number of results obtained in PubMed \\
\hline PEDro & 430 \\
\hline \#"Stroke" "virtual reality" "gait" "balance" & Number of results obtained in PEDro \\
\hline & 24 \\
\hline Scopus & \\
\hline \#1 Tittle-Abs ("Stroke") & \\
\#2 Tittle-Abs ("Virtual reality") \\
\#3 Tittle-Abs ("video games") \\
\#4 \#2 OR \#3 \\
\#5 Tittle-Abs ("Physical Therapy") \\
\#6 Tittle-Abs ("Rehabilitation") \\
\#7 \#5 OR \#6 \\
\#8 Tittle-Abs ("Balance") \\
\#9 Tittle-Abs ("Gait") \\
\#10 Tittle-Abs ("Postural Balance") \\
\#11 Tittle-Abs ("Postural Control") \\
\#12 \#8 OR \#9 OR \#10 OR \#11 \\
\#13 \#1 AND \#4 and \#7 and \#12 \\
\hline
\end{tabular}

Number of results obtained in Scopus

MM: exact major subject heading, MH: exact subject heading, TX: whole text and PEDro: Physiotherapy Evidence Database.

\subsection{Eligibility Criteria}

The eligibility criteria following the Population, Intervention, Comparation and Outcome (PICO) framework is shown in Table 2. 
Table 2. Eligibility criteria according to Population, Intervention, Comparation and Outcome (PICO).

P: adult participants ( $>18$ years of age) from both genders, diagnosed clinically with stroke and gait and/or balance disorders;

I: interventions using commercial video games (Nintendo Wii ${ }^{\circledR}$, Microsoft Xbox ${ }^{\circledR}$, Microsoft Xbox $^{\circledR}$ in combination with Kinect ${ }^{\circledR}$ device or a combination of the previous ones) to treat balance and gait after a stroke; C: studies comparing two exercise interventions (one of them was a programme of VR training and the other was a conventional rehabilitation programme) and

O: provide balance and/or gait results registered with instrumentation systems and/or observational ratings (posturography, static balance index, postural assessment scale, Berg balance scale, Timed Up and Go test, Functional Reach Test, Falls Efficacy Scale International, 10-m walk test, 6-min walk test and dynamic gait index test).

The included studies were prospective controlled clinical trials. While studies that included high-cost VR systems or other systems different than commercial video games were excluded.

\subsection{Extracting Information and Managing Data}

The following data were extracted: number and characteristics of participants; types of interventions; protocol applied in terms of time, frequency and number of sessions; outcome results on balance and gait; methodological quality and risk of bias.

\subsection{Assessing the Quality of Evidence}

The methodological quality of each study was assessed by two independent reviewers using Cochrane's standard quality assessment format [23] and using Review Manager software (V.5.3. The Cochrane Collaboration, Copenhagen, Denmark), which includes a description and rating for each item with a table of "Risk of Bias". A third reviewer was there to help with discrepancies. The independent assessment of each item was considered "a risk of bias" when the study adequately addressed each domain. If not enough information was provided to be able to assess it, it was considered "unclear or doubtful".

Additionally, the Physiotherapy Evidence Database (PEDro) Scale, developed by The Centre of Evidence-Based Physiotherapy (CEBP), was used to assess the methodological quality of each paper. The PEDro Scale, a 10-item scale, is a validated, reliable and versatile tool used to rate randomised controlled trials (RCTs). It has been used as a measurement of methodological quality in many systematic and literature reviews.

\section{Results}

A total of 7648 studies were initially identified. After excluding duplicates, 674 were sieved by title and abstract, and 637 studies were ruled out, since they did not satisfy the eligibility criteria. An additional 20 references were obtained through inverse searches based on the 37 previously selected studies, which were potentially relevant due to the title and abstract. The 57 selected studies by title and abstract were read in-depth: 39 of them were excluded, since they did not satisfy the eligibility criteria. Finally, 18 papers were included in this SR [25-42] (Figure 1 and Supplementary Materials). 


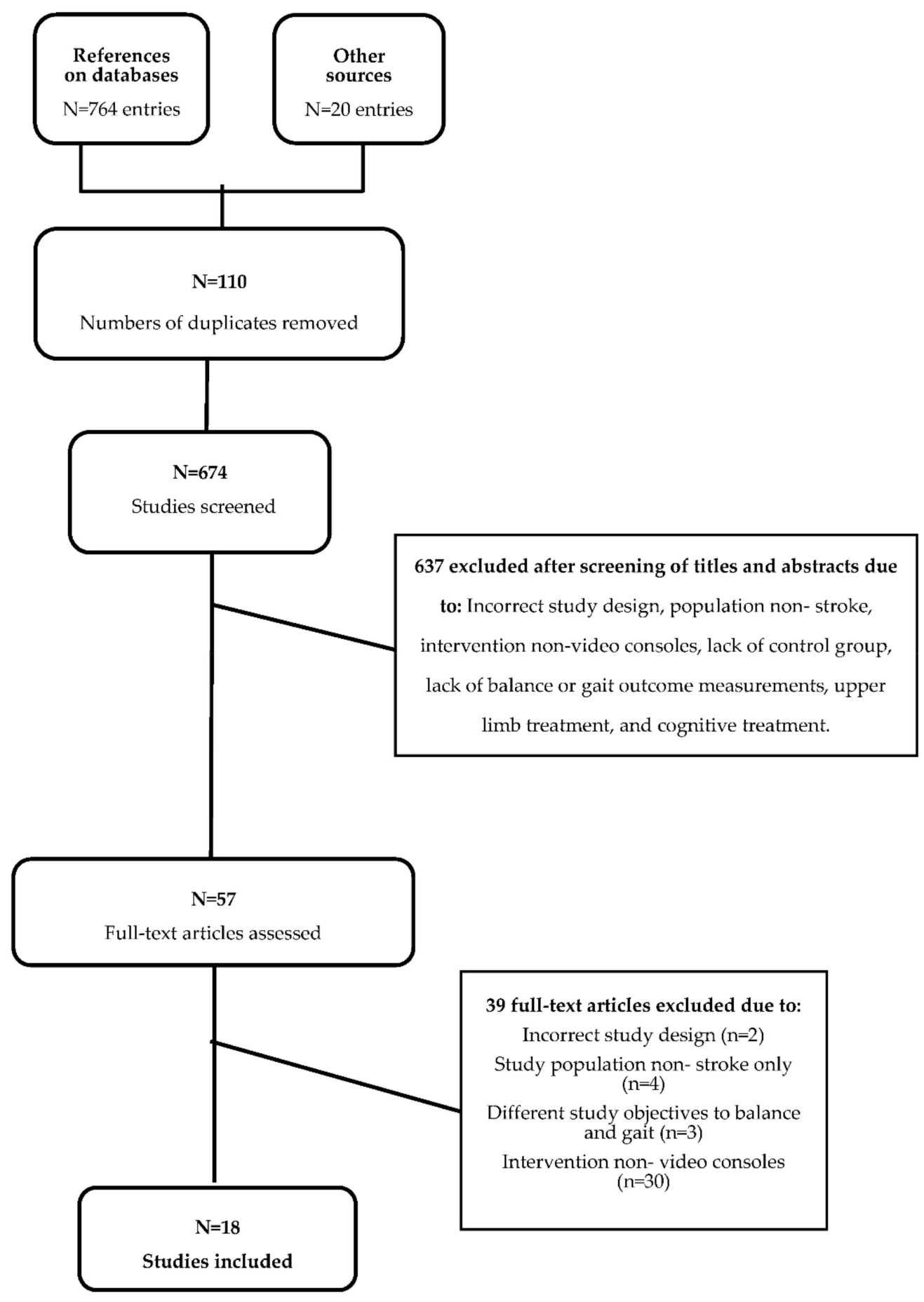

Figure 1. Flow chart.

\subsection{Characteristics of the Included Studies}

\subsubsection{Participants}

A total of 479 individuals participated in the 18 studies included in this SR, 247 of whom performed the experimental intervention and 232 the control intervention. The mean of patients for each study was $26.61 \pm 10.21$ (mean \pm standard deviation), with an average age of $58.92 \pm 6.47$ years in the experimental group (EG), and in the control group (CG), the average ages were $58.17 \pm 6.83$ years and $59.66 \pm 6.20$ years, respectively. Sixteen of the 18 studies collected information on the gender of 
participants, where the distribution was $55.04 \%$ men and 44.95\% women [25-31,33-35,37-42]. In 12 of the studies, the type of stroke was defined, with $66.19 \%$ of the cases being ischaemic heart attacks and $33.8 \%$ brain haemorrhage [25-32,34,35,37,39]. Fourteen studies specified where the stroke was located: $49.62 \%$ were in the right hemisphere, and $50.37 \%$ were in the left hemisphere $[25-27,29,30,34-42]$. The average time since the stroke was $24.23 \pm 27.26$ months. Only two studies did not include this data $[31,33]$. In four studies, the participants were in an acute and/or subacute state $[26,35,36,39]$; in one study, the participants had suffered a stroke within the last 12 months [31], and in the other 13 studies, the participants were in a chronic state.

\subsubsection{Intervention}

Regarding the commercial video games systems used, 13 studies compared the results on balance using a video game programme with the Nintendo Wii ${ }^{\circledR}$ (Nintendo Co., Ltd., Kyoto, Japan) console compared to other rehabilitation treatments $[25-33,35,36,38,42]$, whereby the software Wii Fit $^{\circledR}$ (Nintendo Co., Ltd., Kyoto, Japan) was used in five of them $[25,27,30,31,36]$, and Wii Fit Plus ${ }^{\circledR}$ (Nintendo Co., Ltd., Kyoto, Japan) was used in the other five [26,28,29,33,42]. Both commercial software required the use of the Wii Balance Board ${ }^{\circledR}$ (Nintendo Co., Ltd., Kyoto, Japan) for some games based on the fact that the player has to stay in the limits of the table and work on changing the weight, transferring loads from one foot to another and/or back and forth. Wii Sports ${ }^{\circledR}$ software (Nintendo Co., Ltd., Kyoto, Japan) was used in two papers [32,38] and Wii Sports Resort in one [35]; these two video games do not limit the playing space, and the player can practice some sports (tennis, boxing, golf, etc.) using the $\mathrm{Wii}^{\circledR}$ controllers in one or both hands, as required in each of the video games. In them, the players also practice the transfer of loads. In addition, displacement in different directions is necessary.

Along the same lines, three studies analysed the effects of using the Kinect-Xbox Microsoft ${ }^{\circledR}$ (Microsoft Inc., Redmond, WA, USA) console compared to an outpatient physiotherapy programme [34, 37,41], which used Kinect Sport ${ }^{\circledR}$, Kinect Sport Season $2^{\circledR}[34,37,41]$, Kinect Adventures ${ }^{\circledR}[34,41]$, Kinect Grunstringer ${ }^{\circledR}[41]$ and Kinect Your Shape: Fitness Evolved ${ }^{\circledR}$ [37] (Microsoft Inc., Redmond, WA, USA). In all of them, the player does not need controllers, and the playing space is not limited. Load transfers, displacement and even jumps are practiced in them. In two papers, they studied, on the one hand, the use of the Nintendo Wii ${ }^{\circledR}$ or Kinect-Xbox Microsoft ${ }^{\circledR}$ console [39] using Wii Fit ${ }^{\circledR}$ and Kinect Adventures ${ }^{\circledR}$ software, and on the other hand, its combined use [40] using Wii Fit Plus ${ }^{\circledR}$ and Kinect Adventures ${ }^{\circledR}$ softwares. All these studies used commercial softwares.

As regards the therapy performed by the CG in the different studies, one of them involved using the MOTOmed ergometric bike [41]; in nine studies, only group or individual conventional physiotherapy was used $[25,27,30,31,35,37-40]$; in the rest of the studies, conventional rehabilitation was also used plus another therapy —one added a VR system treatment using the Wii ${ }^{\circledR}$ (but in a sitting position and with different software than that used in the EG), Wii Sports ${ }^{\circledR}$ and Wii Sports Resort ${ }^{\circledR}$ [26]; in five studies, balance treatments were included [28,29,34,36,42]; in another study, a task-oriented treatment was included [33] and, in another study, electrical stimulation of the tibialis anterior was included [32]. None of the studies showed differences between the EG and CG regarding the number of sessions, frequency and length of the intervention. However, in five studies, differences in the session length existed, with the EG receiving 30 min more treatment per session [25,27,31,32,37] (Table 3). 
Table 3. Main characteristics of the studies included in the systematic review (SR) and PEDro.

\begin{tabular}{|c|c|c|c|c|c|c|c|c|c|c|}
\hline Study & Sample Size & $\begin{array}{c}\text { Mean Age } \pm \\
\text { SD }\end{array}$ & $\begin{array}{c}\text { EG } \\
\text { Intervention }\end{array}$ & $\begin{array}{c}\mathbf{N}^{\circ} \text { of } \\
\text { Sessions }\end{array}$ & Sessions/Week & $\begin{array}{c}\text { Duration } \\
\text { (Weeks) }\end{array}$ & $\begin{array}{c}\text { CG } \\
\text { Intervention }\end{array}$ & $\begin{array}{c}\text { Video } \\
\text { Console }\end{array}$ & $\begin{array}{c}\text { Video Games in } \\
\text { EG }\end{array}$ & PEDro \\
\hline $\begin{array}{c}\text { Barcala et al. } \\
\text { [25] }\end{array}$ & $\begin{array}{c}N=20 \\
E G=10 \\
C G=10\end{array}$ & $64.35 \pm 1.20$ & $\begin{array}{c}60^{\prime} \\
\text { conventional } \\
\text { physiotherapy } \\
+30^{\prime} \text { Wii }^{\circledR}\end{array}$ & 10 & 2 & 5 & $\begin{array}{c}60^{\prime} \\
\text { conventional } \\
\text { physiotherapy }\end{array}$ & $\begin{array}{l}\text { Nintendo } \\
\text { Wii }^{\circledR}\end{array}$ & Wii Fit ${ }^{\circledR}$ & $8 / 10$ \\
\hline $\begin{array}{c}\text { Bower et al. } \\
\text { [26] }\end{array}$ & $\begin{array}{l}\mathrm{N}=30 \\
\mathrm{EG}=17 \\
\mathrm{CG}=13\end{array}$ & $63.9 \pm 0.82$ & $\begin{array}{l}\text { Conventional } \\
\text { rehabilitation } \\
+45^{\prime} \mathrm{Wii}^{\circledR} \text { in } \\
\text { bipedestations }\end{array}$ & 9 & 3 & 3 & 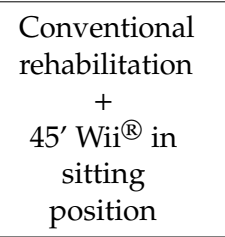 & $\begin{array}{l}\text { Nintendo } \\
\text { Wii }^{\circledR}\end{array}$ & Wii Fit Plus ${ }^{\circledR}$ & $6 / 10$ \\
\hline $\begin{array}{l}\text { Cho et al. } \\
\text { [27] }\end{array}$ & $\begin{array}{l}N=22 \\
E G=11 \\
C G=11\end{array}$ & $64.19 \pm 1.50$ & $\begin{array}{l}60^{\prime} / 90^{\prime} \text { standard } \\
\text { rehabilitation } \\
+30^{\prime} \mathrm{Wii}^{\circledR}\end{array}$ & 18 & 3 & 6 & $\begin{array}{c}60^{\prime} / 90^{\prime} \\
\text { standard } \\
\text { rehabilitation }\end{array}$ & $\begin{array}{l}\text { Nintendo } \\
\text { Wii }^{\circledR}\end{array}$ & Wii Fit ${ }^{\circledR}$ & $6 / 10$ \\
\hline $\begin{array}{l}\text { Hung et al. } \\
\text { [28] }\end{array}$ & $\begin{array}{c}\mathrm{N}=24 \\
\mathrm{EG}=12 \\
\mathrm{CG}=12\end{array}$ & & $\begin{array}{l}\text { Standard } \\
\text { rehabilitation } \\
+30^{\prime} \mathrm{Wii}^{\circledR}\end{array}$ & 24 & 2 & 12 & $\begin{array}{l}\text { Standard } \\
\text { rehabilitation } \\
+30^{\prime} \text { load } \\
\text { transfer }\end{array}$ & $\begin{array}{l}\text { Nintendo } \\
\text { Wii }^{\circledR}\end{array}$ & Wii Fit Plus ${ }^{\circledR}$ & $8 / 10$ \\
\hline $\begin{array}{l}\text { Hung et al. } \\
\text { [29] }\end{array}$ & $\begin{array}{c}N=28 \\
E G=13 \\
C G=15\end{array}$ & $54.39 \pm 1.40$ & $\begin{array}{l}\text { Standard } \\
\text { outpatient } \\
\text { rehabilitation } \\
+30^{\prime} \mathrm{Wii}^{\circledR}\end{array}$ & 24 & 2 & 12 & $\begin{array}{c}\text { Standard } \\
\text { outpatient } \\
\text { rehabilitation } \\
+30^{\prime} \text { load } \\
\text { transfer }\end{array}$ & $\begin{array}{l}\text { Nintendo } \\
\text { Wii }^{\circledR}\end{array}$ & Wii Fit Plus ${ }^{\circledR}$ & $8 / 10$ \\
\hline $\begin{array}{c}\text { Kannan et al. } \\
\text { [30] }\end{array}$ & $\begin{array}{c}\mathrm{N}=20 \\
\mathrm{EG}=10 \\
\mathrm{CG}=10\end{array}$ & $59.25 \pm 2.47$ & $\begin{array}{c}90^{\prime} \mathrm{Wii}^{\circledR}+ \\
\text { cognitive } \\
\text { tasks } \\
\text { simultaneously }\end{array}$ & 20 & & 6 & $\begin{array}{l}90^{\prime} \text { standard } \\
\text { rehabilitation }\end{array}$ & $\begin{array}{l}\text { Nintendo } \\
\text { Wii }^{\circledR}\end{array}$ & Wii Fit ${ }^{\circledR}$ & $6 / 10$ \\
\hline $\begin{array}{c}\text { Karasu et al. } \\
{[31]}\end{array}$ & $\begin{array}{l}\mathrm{N}=23 \\
\mathrm{EG}=12 \\
\mathrm{CG}=11\end{array}$ & $63.2 \pm 1.27$ & $\begin{array}{l}120^{\prime} / 180^{\prime} \text { standard } \\
\text { rehabilitation } \\
+20^{\prime} \mathrm{Wii}^{\circledR}\end{array}$ & 20 & 5 & 4 & $\begin{array}{l}120^{\prime} / 180^{\prime} \text { standar } \\
\text { rehabilitation }\end{array}$ & $\begin{array}{l}\text { dNintendo } \\
\text { Wii }{ }^{\circledR}\end{array}$ & Wii Fit ${ }^{\circledR}$ & $7 / 10$ \\
\hline
\end{tabular}


Table 3. Cont

\begin{tabular}{|c|c|c|c|c|c|c|c|c|c|c|}
\hline Study & Sample Size & $\begin{array}{c}\text { Mean Age } \pm \\
\text { SD }\end{array}$ & $\begin{array}{c}\text { EG } \\
\text { Intervention }\end{array}$ & $\begin{array}{c}\mathbf{N}^{\circ} \text { of } \\
\text { Sessions }\end{array}$ & Sessions/Week & $\begin{array}{l}\text { Duration } \\
\text { (Weeks) }\end{array}$ & $\begin{array}{c}\text { CG } \\
\text { Intervention }\end{array}$ & $\begin{array}{c}\text { Video } \\
\text { Console } \\
\end{array}$ & $\begin{array}{c}\text { Video Games in } \\
\text { EG }\end{array}$ & PEDro \\
\hline $\begin{array}{c}\text { Kim et al. } \\
\text { [32] }\end{array}$ & $\begin{array}{c}N=17 \\
E G=10 \\
C G=7\end{array}$ & $48.15 \pm 9.68$ & $\begin{array}{l}30^{\prime} \text { general } \\
\text { exercises + } \\
15^{\prime} \text { electric } \\
\text { stimulation } \\
+30^{\prime} \mathrm{Wii}^{\circledR}\end{array}$ & 9 & 3 & 3 & $\begin{array}{l}30^{\prime} \text { general } \\
\text { exercises }+ \\
15^{\prime} \text { electric } \\
\text { stimulation }\end{array}$ & $\begin{array}{l}\text { Nintendo } \\
\mathrm{Wii}^{\circledR}\end{array}$ & Wii Sports ${ }^{\circledR}$ & $6 / 10$ \\
\hline $\begin{array}{l}\text { Lee et al. } \\
\text { [33] }\end{array}$ & $\begin{array}{c}N=24 \\
E G=12 \\
C G=12\end{array}$ & $47.53 \pm 2.29$ & $\begin{array}{l}60^{\prime} \\
\text { therapeutic } \\
\text { exercise with } \\
\text { physiotherapy } \\
+30^{\prime} \text { Wii }{ }^{\circledR}\end{array}$ & 18 & 3 & 6 & $\begin{array}{c}60^{\prime} \\
\text { therapeutic } \\
\text { exercise }+30^{\prime} \\
\text { functional } \\
\text { task } \\
\text { treatment }\end{array}$ & $\begin{array}{l}\text { Nintendo } \\
\mathrm{Wii}^{\circledR}\end{array}$ & Wii Fit Plus ${ }^{\circledR}$ & $6 / 10$ \\
\hline $\begin{array}{l}\text { Lee et al. } \\
\text { [34] }\end{array}$ & $\begin{array}{l}\mathrm{N}=47 \\
\mathrm{EG}=26 \\
\mathrm{CG}=21\end{array}$ & $57.55 \pm 2.53$ & \begin{tabular}{l}
\multicolumn{1}{c}{$45^{\prime}$} \\
conventional \\
rehabilitation \\
$+45^{\prime} \mathrm{Xbox}^{\circledR}$
\end{tabular} & 12 & 2 & 6 & $\begin{array}{l}60^{\prime} \\
\text { conventional } \\
\text { rehabilitation } \\
+30^{\prime} \text { balance } \\
\text { training }\end{array}$ & Kinect-Xbox ${ }^{\circledR}$ & $\begin{array}{c}\text { Kinect Sport }{ }^{\circledR}+ \\
\text { Kinect Sport } \\
\text { Season } 2^{\circledR}+ \\
\text { Kinect } \\
\text { Adventures }{ }^{\circledR}+ \\
\text { Kinect Your Shape: } \\
\text { Fitness Evolved }{ }^{\circledR}\end{array}$ & $7 / 10$ \\
\hline $\begin{array}{l}\text { Lee et al. } \\
\text { [35] }\end{array}$ & $\begin{array}{l}N=10 \\
E G=5 \\
C G=5\end{array}$ & $65.7 \pm 0.5$ & $\begin{array}{l}135^{\prime} \\
\text { conventional } \\
\text { rehabilitation } \\
+30^{\prime} \mathrm{Wii}^{\circledR}\end{array}$ & 12 & 3 & 4 & $\begin{array}{c}135^{\prime} \\
\text { conventional } \\
\text { rehabilitation }\end{array}$ & $\begin{array}{l}\text { Nintendo } \\
\text { Wii }^{\circledR}\end{array}$ & $\begin{array}{l}\text { Wii Sports } \\
\text { Resort }^{\circledR}\end{array}$ & $7 / 10$ \\
\hline $\begin{array}{l}\text { Morone et al. } \\
\text { [36] }\end{array}$ & $\begin{array}{l}\mathrm{N}=50 \\
\mathrm{EG}=25 \\
\mathrm{CG}=25\end{array}$ & $60.16 \pm 10.04$ & $\begin{array}{l}\text { Conventional } \\
\text { physiotherapy } \\
+20^{\prime} \mathrm{Wii}^{\circledR}\end{array}$ & 12 & 3 & 4 & $\begin{array}{l}\text { Conventional } \\
\text { physiotherapy } \\
+20^{\prime} \text { balance } \\
\text { exercises }\end{array}$ & $\begin{array}{l}\text { Nintendo } \\
\mathrm{Wii}^{\circledR}\end{array}$ & Wii Fit ${ }^{\circledR}$ & $8 / 10$ \\
\hline $\begin{array}{l}\text { Park et al. } \\
\text { [37] }\end{array}$ & $\begin{array}{l}N=20 \\
E G=10 \\
C G=10\end{array}$ & $63.65 \pm 2.33$ & \begin{tabular}{l}
\multicolumn{1}{c}{$30^{\prime}$} \\
conventional \\
physiotherapy \\
$+30^{\prime}$ Xbox $^{\circledR}$
\end{tabular} & 42 & 7 & 6 & $\begin{array}{l}\text { 30' } \\
\text { conventional } \\
\text { physiotherapy }\end{array}$ & Kinect-Xbox ${ }^{\circledR}$ & $\begin{array}{l}\text { Kinect Sport }^{\circledR}+ \\
\text { Kinect Sport } \\
\text { Season } 2^{\circledR}\end{array}$ & $8 / 10$ \\
\hline
\end{tabular}


Table 3. Cont

\begin{tabular}{|c|c|c|c|c|c|c|c|c|c|c|}
\hline Study & Sample Size & $\begin{array}{c}\text { Mean Age } \pm \\
\text { SD }\end{array}$ & $\begin{array}{c}\text { EG } \\
\text { Intervention }\end{array}$ & $\begin{array}{c}\mathbf{N}^{\circ} \text { of } \\
\text { Sessions }\end{array}$ & Sessions/Week & $\begin{array}{c}\text { Duration } \\
\text { (Weeks) }\end{array}$ & $\begin{array}{c}\text { CG } \\
\text { Intervention }\end{array}$ & $\begin{array}{c}\text { Video } \\
\text { Console }\end{array}$ & $\begin{array}{c}\text { Video Games in } \\
\text { EG }\end{array}$ & PEDro \\
\hline $\begin{array}{c}\text { Pedreira da } \\
\text { Fonseca et al. } \\
\text { [38] }\end{array}$ & $\begin{array}{l}N=27 \\
E G=14 \\
C G=13\end{array}$ & $52.4 \pm 8.9$ & $\begin{array}{c}15^{\prime} \\
\text { conventional } \\
\text { physiotherapy } \\
+45^{\prime} \mathrm{Wii}^{\circledR}\end{array}$ & 20 & 2 & 10 & $\begin{array}{l}60^{\prime} \\
\text { conventional } \\
\text { physiotherapy }\end{array}$ & $\begin{array}{l}\text { Nintendo } \\
\text { Wii }^{\circledR}\end{array}$ & Wii Sports ${ }^{\circledR}$ & $7 / 10$ \\
\hline $\begin{array}{l}\text { Rajaratnam } \\
\text { et al. [39] }\end{array}$ & $\begin{array}{l}N=19 \\
E G=10 \\
C G=9\end{array}$ & $61.6 \pm 7.8$ & $\begin{array}{c}40^{\prime} \\
\text { conventional } \\
\text { rehabilitation } \\
+20^{\prime} \mathrm{VR} \\
\left(\mathrm{Wii}^{\circledR} / \mathrm{Xbox}^{\circledR}\right)\end{array}$ & 15 & & & $\begin{array}{c}60^{\prime} \\
\text { conventional } \\
\text { rehabilitation }\end{array}$ & $\begin{array}{l}\text { Nintendo } \\
\text { Wii }^{\circledR} / \text { Kinect- } \mathrm{Xb}\end{array}$ & $\begin{array}{l}\text { Wii Fit }{ }^{\circledR} / \text { Kinect }^{-} \\
\text {ox }{ }^{\circledR} d \text { ventures }{ }^{\circledR}\end{array}$ & $7 / 10$ \\
\hline $\begin{array}{l}\text { Singh et al. } \\
\text { [40] }\end{array}$ & $\begin{array}{l}N=28 \\
E G=15 \\
C G=13\end{array}$ & $66.2 \pm 1.13$ & $\begin{array}{l}90^{\prime} \text { standard } \\
\text { group } \\
\text { physiotherapy } \\
+30^{\prime} \text { VR }\left(15^{\prime}\right. \\
\mathrm{Wii}^{\circledR}+15^{\prime} \\
\left.\text { Xbox }^{\circledR}\right) \\
\end{array}$ & 12 & 2 & 6 & $\begin{array}{c}120^{\prime} \\
\text { standard } \\
\text { group } \\
\text { physiotherapy }\end{array}$ & $\begin{array}{c}\text { Nintendo } \\
\text { Wii }^{\circledR}+ \\
\text { Kinect-Xbox }^{\circledR}\end{array}$ & $\begin{array}{c}\text { Wii Fit Plus }{ }^{\circledR}+ \\
\text { Kinect } \\
\text { Adventures }\end{array}$ & $5 / 10$ \\
\hline $\begin{array}{c}\text { Song \& Park } \\
\text { [41] }\end{array}$ & $\begin{array}{l}\mathrm{N}=40 \\
\mathrm{EG}=20 \\
\mathrm{CG}=20\end{array}$ & $50.73 \pm 0.89$ & $30^{\prime} \mathrm{Xbox}^{\circledR}$ & 40 & 5 & 8 & $\begin{array}{c}30^{\prime} \\
\text { ergometric } \\
\text { bike } \\
\text { MOTOmed }\end{array}$ & Kinect-Xbox ${ }^{\circledR}$ & $\begin{array}{c}\text { Kinect Sport }^{\circledR}+ \\
\text { Kinect Sport } \\
\text { Season } 2^{\circledR}+ \\
\text { Kinect } \\
\text { Adventures }^{\circledR}+ \\
\text { Kinect } \\
\text { Grunstringer } \\
\text { G }\end{array}$ & $6 / 10$ \\
\hline $\begin{array}{c}\text { Yatar \& } \\
\text { Yildrim [42] }\end{array}$ & $\begin{array}{l}N=30 \\
E G=15 \\
C G=15\end{array}$ & $59.7 \pm 4.38$ & $\begin{array}{l}30^{\prime} \\
\text { Neurodevelopment } \\
+30^{\prime} \mathrm{Wii}^{\circledR}\end{array}$ & nt 12 & 3 & 4 & $\begin{array}{l}30^{\prime} \\
\text { Neurodevelopr } \\
+30^{\prime} \text { balance } \\
\text { training }\end{array}$ & $\begin{array}{l}\text { mentintendo } \\
\text { Wii }^{\circledR}\end{array}$ & Wii Fit Plus ${ }^{\circledR}$ & $4 / 10$ \\
\hline
\end{tabular}




\subsubsection{Outcomes}

In 12 out of 18 studies, the balance of participants in bipedestations was analysed [25-27,29-33,39-42], in seven studies, gait was analysed [30,36-38,40-42] and, in 17 studies, aspects related to balance during functional tasks were analysed [25-37,39-42].

Data related to maintaining bipedestations were registered using instrumentation systems, such as the Wii Balance Board ${ }^{\circledR}[26,33,39,42]$, pressure disc $[25,40]$ and dynamometric platforms $[27,29-31,41]$. Only four studies used the Steptest observational tests [26], the Static Balance Index (SBI) [31] and Postural Assessment Scale (PASS) [31,32].

In order to assess balance during functional tasks, the following observational scales were used: the Berg Balance Scale (BBS) in 11 studies [27,28,30-32,34-37,39,42], the Timed Up and Go test (TUG) in 12 studies [25-27,29-31,35,37,39-42], the Functional Reach Test (FRT) in eight studies [26,29,31,33-35,39,42] and the Falls Efficacy Scale International (FES-I) in two studies [26,29].

On the other hand, the variables related to gait were registered in four studies using the 10-metre walk test (10 MWT) $[36,37,40,42]$, the 6-min walk test (6 MWT) [30,40] and the Dynamic Gait Index (DGI) $[38,42]$.

Four studies included possible adverse effects derived from the intervention [26,29,34,37], which only were evidenced in three of them $[26,29,34]$. In one study, four EG and two CG participants experienced a fall during treatment, but they were inconsequential. Those in the EG also manifested an increase in pain, more so than those in the CG; however, this pain did not last more than $24 \mathrm{~h}$. Likewise, an intergroup difference existed as regards the sensation of fatigue experienced by the EG participants in comparison to the CG participants after each session [26]. In other study, two CG participants reported increased knee pain when training, and three EG participants reported increased spasticity when playing with the Wii Balance Board ${ }^{\circledR}$, but this symptom subsided when they played other games [29]. In the other one, both groups experienced pain, hypertonia and dizziness and were greater in the EG compared to the CG [34].

\subsubsection{Main Results}

Regarding balance in bipedestations, in four of the included studies, no changes were observed after the experimental intervention or after the comparison [27,39,40,42]. In four studies, significant statisticaldifferences were found regarding pre-post-treatments in each group but not between both groups $[29,30,33,43]$. On the other hand, in three studies, the results showed significant statistical differences as regards pre-post-treatments in each group and between both groups [31,32,41], and in one of them, differences in each group and differences between groups in the follow-up assessment were evidenced [26]. As regards balance during functional tasks, significant statistical differences were found in pre-post-treatments in each group [25-30,32-37,39-42], and differences between groups were evidenced in nine of them in favour of the experimental treatment [27,31,33,35-37,39,41,42].

For gait, one of the studies showed no pre-post changes in the two intervention groups [40]. In two studies, significant differences were found as regards pre-post-treatments in each group but with no differences between groups [30,42]. In one study, significant differences were found only in the CG after treatment [38]. In the other three studies, significant statistical differences were found relating to pre-post-treatments in each group and between both groups in favour of the experimental treatment $[36,37,41]$ (Table 4). The effect size was only showed in Bower et al. [26]. 
Table 4. Results of the studies included in the SR.

\begin{tabular}{|c|c|c|c|c|c|c|}
\hline Study & Outcomes & $\begin{array}{c}\text { Mean Values EG } \\
(\text { Mean } \pm \text { SD })\end{array}$ & $\begin{array}{l}\text { Mean Values CG } \\
\quad(\text { Mean } \pm \text { SD) }\end{array}$ & Main Results & $\begin{array}{c}\text { Effect Size } \\
\text { (Cohen's d) }\end{array}$ & $\begin{array}{c}\text { Time } \times \text { Group } \\
\text { Interaction } \\
(\mathrm{F} / \mathrm{P})\end{array}$ \\
\hline Barcala et al. [25] & $\begin{array}{l}\text { Posturography (peak } \\
\text { plantar pressure and } \\
\text { oscillation in different } \\
\text { sensorial conditions) } \\
\text { BBS } \\
\text { TUG }\end{array}$ & $\begin{array}{l}\text { Peak plantar pressure } \\
\text { on affected leg, g/cm²: } \\
\text { pre: } 1138.2 \pm 333.3 ; \\
\text { post: } 1309.5 \pm 260.1 ; \\
p<0.05 \\
\text { Peak plantar pressure } \\
\text { on non-affected leg, } \\
\text { g/cm }{ }^{2}: \\
\text { pre: } 1465.7 \pm 285.2 ; \\
\text { post: } 1350.4 \pm 243.6 ; \\
p<0.05 \\
\text { ML oscillation EO, } \\
\text { cm: } \\
\text { pre: } 4.55 \pm 0.55 ; \\
\text { post:2.87 } \pm 0.93 ; \\
p<0.05 \\
\text { ML oscillation EC, cm: } \\
\text { pre: } 5.40 \pm 0.51 ; \\
\text { post: } 3.97 \pm 0.94 ; \\
p<0.05 \\
\text { AP oscillation EO, cm: } \\
\text { pre: } 1.18 \pm 0.06 ; \\
\text { post: } 1.13 \pm 0.08 ; \\
p<0.05 \\
\text { AP oscillation EC, cm: } \\
\text { pre: } 1.48 \pm 0.27 ; \\
\text { post: } 1.35 \pm 0.27 ; \\
p<0.05 \\
\text { BBS, score: } \\
\text { Pre: } 39.6 \pm 6.43 ; \\
\text { post: } 41.9 \pm 6.91 ; \\
p<0.05 \\
\text { TUG, s: } \\
\text { Pre: } 27.9 \pm 8.22 ; \\
\text { post: } 24.3 \pm 8.64 ; \\
p<0.05\end{array}$ & $\begin{array}{l}\text { Peak plantar pressure } \\
\text { on affected leg, g/cm²: } \\
\text { pre: } 1232.1 \pm 182.1 \\
\text { post: } 1319.7 \pm 194.6 \\
p<0.05 \\
\text { Peak plantar pressure } \\
\text { on non-affected leg, } \\
\text { g/cm }{ }^{2}: \\
\text { pre: } 1490.9 \pm 196.1 ; \\
\text { post: } 1357.0 \pm 185.4 ; \\
p<0.05 \\
\text { ML oscillation EO, } \\
\text { cm: pre: } 4.3 \pm 1.69 ; \\
\text { post: } 3.13 \pm 1.81 ; \\
p<0.05 \\
\text { ML oscillation EC, cm: } \\
\text { pre: } 5.17 \pm 2.07 ; \\
\text { post: } 3.64 \pm 1.97 ; \\
p<0.05 \\
\text { AP oscillation EO, cm: } \\
\text { pre: } 1.18 \pm 0.13 ; \\
\text { post: } 1.12 \pm 0.08 ; \\
p<0.05 \\
\text { AP oscillation EC, cm: } \\
\text { pre: } 1.26 \pm 0.15 ; \\
\text { post: } 1.18 \pm 0.07 ; \\
p<0.05 \\
\text { BBS, score: } \\
\text { Pre: } 37.2 \pm 5.22 \\
\text { post: } 42.2 \pm 4.80 \\
p<0.05 \\
\text { TUG, s: } \\
\text { Pre: } 28.1 \pm 3.10 ; \\
\text { post: } 25.2 \pm 2.78 ; \\
p<0.05\end{array}$ & $\begin{array}{l}\text { } \\
\\
\text { Statistically } \\
\text { significant } \\
\text { improvements in both } \\
\text { groups after } \\
\text { intervention in all the } \\
\text { variables (the } \\
\text { variables recorded by } \\
\text { posturography, } \\
\text { BBS and TUG) ( } p< \\
\text { 0.05), } \\
\text { although without } \\
\text { significant statistical } \\
\text { differences between } \\
\text { groups. }\end{array}$ & NR & NR \\
\hline
\end{tabular}


Table 4. Cont.

\begin{tabular}{|c|c|c|c|c|c|c|}
\hline Study & Outcomes & $\begin{array}{l}\text { Mean Values EG } \\
(\text { Mean } \pm \text { SD) }\end{array}$ & $\begin{array}{l}\text { Mean Values CG } \\
\quad(\text { Mean } \pm \text { SD) }\end{array}$ & Main Results & $\begin{array}{l}\text { Effect Size } \\
\text { (Cohen's d) }\end{array}$ & $\begin{array}{c}\text { Time } \times \text { Group } \\
\text { Interaction } \\
(\mathrm{F} / \mathrm{P})\end{array}$ \\
\hline Bower et al. [26] & $\begin{array}{l}\text { Wii Balance Board }{ }^{\circledR} \\
\text { (displacement } \\
\text { velocity in different } \\
\text { sensorial conditions } \\
\text { and weight shifting) } \\
\text { Steptest } \\
\text { FRT } \\
\text { TUG } \\
\text { FES-I }\end{array}$ & $\begin{array}{l}\text { EO COP velocity, } \\
\text { cm/s: } \\
\text { pre: } 1.90 \pm 0.76 ; \\
\text { post: } 1.59 \pm 0.49 ; \\
p>0.05 \\
\text { EO ML COP velocity, } \\
\text { cm/s: } \\
\text { pre: } 0.94 \pm 0.45 ; \\
\text { post: } 0.71 \pm 0.27 ; \\
p>0.05 \mathrm{EO} \text { AP } \\
\text { velocity, cm/s: } \\
\text { Pre: } 1.45 \pm 0.60 ; \\
\text { post: } 1.28 \pm 0.41 ; \\
p>0.05 \\
\text { EC COP velocity, } \\
\text { cm/s: } \\
\text { pre: } 2.89 \pm 1.30 ; \\
\text { post: } 2.29 \pm 0.70 ; \\
p>0.05 \\
\text { EC ML COP velocity, } \\
\text { cm/s: } \\
\text { pre: } 1.38 \pm 0.99 ; \\
\text { post: } 0.94 \pm 0.38 ; \\
p>0.05 \\
\text { EC AP velocity, cm/s: } \\
\text { pre: } 2.21 \pm 0.84 ; \\
\text { post: } 1.91 \pm 0.60 ; \\
p>0.05 \\
\text { ML weight shifting, n: } \\
\text { pre: } 7.2 \pm 3.5 ; \\
\text { post: } 10.9 \pm 4.0 ; \\
p>0.05 \\
\text { Steptest (affected), } \\
\text { score: } \\
\text { pre: } 2.7 \pm 3.8 ; \\
\text { post: } 8.9 \pm 5.4 ; \\
p>0.05\end{array}$ & $\begin{array}{l}\text { EO COP velocity, } \\
\text { cm/s: } \\
\text { pre: } 1.54 \pm 0.38 ; \\
\text { post: } 1.54 \pm 0.42 ; \\
p>0.05 \\
\text { EO ML COP velocity: } \\
\text { pre: } 0.60 \pm 0.13 ; \\
\text { post: } 0.59 \pm 0.16 ; \\
p>0.05 \\
\text { EO AP velocity, cm/s: } \\
\text { Pre: } 1.29 \pm 0.33 ; \\
\text { post: } 1.31 \pm 0.38 ; \\
p>0.05 \\
\text { EC COP velocity, } \\
\text { cm/s: } \\
\text { pre: } 2.62 \pm 0.82 ; \\
\text { post: } 2.36 \pm 0.67 ; \\
p>0.05 \\
\text { EC ML COP velocity, } \\
\text { cm/s: } \\
\text { pre: } 0.89 \pm 0.34 ; \\
\text { post: } 0.76 \pm 0.22 ; \\
p>0.05 \\
\text { EC AP velocity, cm/s: } \\
\text { pre: } 2.27 \pm 0.70 ; \\
\text { post: } 2.12 \pm 0.61 ; \\
p>0.05 \\
\text { ML weight shifting, n: } \\
\text { pre: } 8.1 \pm 3.4 ; \\
\text { post: } 10.1 \pm 2.3 ; \\
p>0.05 \\
\text { Steptest (affected), } \\
\text { score: } \\
\text { pre: } 7.4 \pm 6.0 ; \\
\text { post: } 8.9 \pm 5.4 ; \\
p>0.05\end{array}$ & $\begin{array}{c}\text { Clinical } \\
\text { improvements in both } \\
\text { groups after } \\
\text { intervention in all the } \\
\text { variables (the } \\
\text { variables recorded by } \\
\text { Wii Balance Board }{ }^{\circledR} \text {, } \\
\text { Steptest, FRT, TUG, } \\
\text { FES-I), but not } \\
\text { statistically significant } \\
\text { improvements ( } p> \\
0.05 \text { ). } \\
\text { Not statistic and } \\
\text { clinically significant } \\
\text { differences between } \\
\text { groups in any } \\
\text { variable after } \\
\text { intervention. }\end{array}$ & $\begin{array}{c}\text { EO COP velocity: } \\
-0.93 \\
\text { EO ML COP velocity: } \\
-1 \\
\text { EO AP velocity: }-0.69 \\
\text { EC COP velocity: } \\
-0.46 \\
\text { EC ML COP velocity: } \\
-0.58 \\
\text { EC AP velocity: } \\
-0.30 \\
\text { ML weight shifting: } \\
0.71 \\
\text { Steptest (affected): } \\
1.54 \\
\text { Steptest (unaffected): } \\
0.41 \\
\text { FRT: } 0.03 \\
\text { TUG: } 0.29 \\
\text { FES-I: } 0.19\end{array}$ & $\begin{array}{c}\text { Steptest NR/0.002 } \\
\text { FRT: NR/0.066 } \\
\text { NR in the other } \\
\text { variables }\end{array}$ \\
\hline
\end{tabular}


Table 4. Cont.

\begin{tabular}{|c|c|c|c|c|c|c|}
\hline Study & Outcomes & $\begin{array}{c}\text { Mean Values EG } \\
(\text { Mean } \pm \text { SD) }\end{array}$ & $\begin{array}{c}\text { Mean Values CG } \\
(\text { Mean } \pm \text { SD) }\end{array}$ & Main Results & $\begin{array}{l}\text { Effect Size } \\
\text { (Cohen's d) }\end{array}$ & $\begin{array}{c}\text { Time } \times \text { Group } \\
\text { Interaction } \\
(\mathrm{F} / \mathrm{P})\end{array}$ \\
\hline & & $\begin{array}{l}\text { Steptest (unaffected), } \\
\text { score: } \\
\text { pre: } 4.2 \pm 3.1 ; \\
\text { post:9.0 } \pm 4.2 ; \\
p>0.05 \\
\text { FRT, cm: } \\
\text { pre: } 28.1 \pm 6.4 ; \\
\text { post: } 31.1 \pm 6.5 ; \\
p>0.05 \\
\text { TUG, s: } \\
\text { Pre: } 30.3 \pm 15.9 ; \\
\text { post: } 19.2 \pm 15.5 ; \\
p>0.05 \\
\text { FES-I, score: } \\
\text { pre: } 16.6 \pm 4.8 ; \\
\text { post: } 11.3 \pm 3.2 ; \\
p>0.05\end{array}$ & $\begin{array}{l}\text { Steptest (unaffected, } \\
\text { score): } \\
\text { pre: } 6.1 \pm 5.8 ; \\
\text { post:9.7 } 94.7 ; \\
p>0.05 \\
\text { FRT, cm: } \\
\text { pre: } 28.3 \pm 9.6 ; \\
\text { post: } 31.1 \pm 9.0 ; \\
p>0.05 \\
\text { TUG, s: } \\
\text { Pre: } 32.0 \pm 32.2 ; \\
\text { Post: } 16.0 \pm 11.9 ; \\
p>0.05 \\
\text { FES-I, score: } \\
\text { pre: } 19.3 \pm 7.0 ; \\
\text { post: } 13.0 \pm 3.4 ; \\
p>0.05\end{array}$ & & & \\
\hline Cho et al. [27] & $\begin{array}{l}\text { Posturography } \\
\text { (Postural Sway } \\
\text { Velocity in different } \\
\text { sensorial conditions) } \\
\text { BBS } \\
\text { TUG }\end{array}$ & $\begin{array}{l}\text { PSV AP EO, mm/s: } \\
\text { Pre: } 7.37 \pm 2.20 ; \\
\text { Post: } 6.20 \pm 1.70 ; \\
p>0.05 \\
\text { PSV AP EC, mm/s: } \\
\text { Pre: } 9.97 \pm 2.69 ; \\
\text { Post:9.18 } \pm 1.75 ; \\
p>0.05 \\
\text { PSV ML EO, mm/s: } \\
\text { Pre: } 11.40 \pm 2.24 ; \\
\text { Post: } 11.22 \pm 2.06 ; \\
p>0.05 \\
\text { PSV ML EC, mm/s: } \\
\text { Pre: } 16.78 \pm 2.25 ; \\
\text { Post: } 15.50 \pm 3.59 ; \\
p>0.05 \\
\text { BBS, score: } \\
\text { Pre: } 39.09 \pm 5.66 ; \\
\text { Post: } 43.09 \pm 4.80 ; \\
p<0.001 \\
\text { TUG, s: } \\
\text { Pre: } 21.74 \pm 3.41 ; \\
\text { Post: } 20.40 \pm 3.19 ; \\
p<0.001\end{array}$ & $\begin{array}{l}\text { PSV AP EO, mm/s: } \\
\text { Pre: } 6.01 \pm 1.85 ; \\
\text { post: } 5.64 \pm 1.57 ; \\
p>0.05 \\
\text { PSV AP EC, mm/s: } \\
\text { Pre: } 9.67 \pm 2.72 ; \\
\text { Post:9.14 } \pm 2.31 ; \\
p>0.05 \\
\text { PSV ML EO, mm/s: } \\
\text { Pre: } 9.92 \pm 1.28 ; \\
\text { Post:9.82 } \pm 1.20 ; \\
p>0.05 \\
\text { PSV ML EC, mm/s: } \\
\text { Pre: } 14.41 \pm 4.08 ; \\
\text { Post: } 14.12 \pm 4.01 ; \\
p>0.05 \\
\text { BBS, score: } \\
\text { Pre: } 41.09 \pm 4.01 ; \\
\text { Post: } 43.90 \pm 4.06 ; \\
p<0.001 \\
\text { TUG, s: } \\
\text { Pre: } 19.60 \pm 4.42 ; \\
\text { Post: } 19.08 \pm 4.52 ; \\
p<0.01\end{array}$ & $\begin{array}{c}\text { } \\
\text { Statistically } \\
\text { significant } \\
\text { improvements in both } \\
\text { groups after } \\
\text { intervention in BBS } \\
\text { and TUG }(p<0.01) \text {; } \\
\text { statistic } \\
\text { improvements were } \\
\text { significantly higher in } \\
\text { the EG ( } p<0.05) . \\
\text { The variables } \\
\text { recorded by } \\
\text { posturography } \\
\text { presented no } \\
\text { significant statistical } \\
\text { differences in any } \\
\text { group after } \\
\text { intervention }(p> \\
0.05) .\end{array}$ & NR & NR \\
\hline
\end{tabular}


Table 4. Cont.

\begin{tabular}{|c|c|c|c|c|c|c|}
\hline Study & Outcomes & $\begin{array}{l}\text { Mean Values EG } \\
\quad(\text { Mean } \pm \text { SD })\end{array}$ & $\begin{array}{l}\text { Mean Values CG } \\
\quad(\text { Mean } \pm \text { SD) }\end{array}$ & Main Results & $\begin{array}{l}\text { Effect Size } \\
\text { (Cohen's d) }\end{array}$ & $\begin{array}{c}\text { Time } \times \text { Group } \\
\text { Interaction } \\
(\mathrm{F} / \mathrm{P})\end{array}$ \\
\hline Hung et al. [28] & BBS & $\begin{array}{l}\text { BBS, score: } \\
\text { Pre: } 46.58 \pm 5.16 \\
\text { Post: } 48.83 \pm 5.02 \\
p>0.05\end{array}$ & $\begin{array}{l}\text { BBS, score: } \\
\text { Pre: } 48.25 \pm 3.91 \\
\text { Post: } 50.42 \pm 3.99 \\
p>0.05\end{array}$ & $\begin{array}{c}\text { Clinical } \\
\text { improvements in both } \\
\text { groups after } \\
\text { intervention in BBS, } \\
\text { but not statistically } \\
\text { significant }(p>0.05) \\
\text { Not significant } \\
\text { statistical differences } \\
\text { between groups in } \\
\text { BBS after } \\
\text { intervention. }\end{array}$ & NR & NR \\
\hline Hung et al. [29] & $\begin{array}{l}\text { Posturography } \\
\text { (Percentage of weight } \\
\text { bearing on affected } \\
\text { leg in different } \\
\text { sensorial conditions, } \\
\text { Stability index in } \\
\text { different sensorial } \\
\text { conditions) } \\
\text { FRT } \\
\text { TUG } \\
\text { FES-I }\end{array}$ & $\begin{array}{l}\text { Weight bearing on } \\
\text { affected leg with head } \\
\text { straight EO while } \\
\text { standing on a solid } \\
\text { surface, \%: } \\
\text { Pre: } 42.31 \pm 7.94 ; \\
\text { Post: } 43.34 \pm 6.03 \text {; } \\
p<0.05 \\
\text { Follow-up: } 42.08 \pm \\
4.00 ; \\
p>0.05 \\
\text { Weight bearing on } \\
\text { affected leg with head } \\
\text { straight EC while } \\
\text { standing on a solid } \\
\text { surface, } \%: \\
\text { Pre: } 43.58 \pm 10.46 ; \\
\text { Post: } 44.03 \pm 6.76 ; \\
P<0.05 \\
\text { Follow-up: } 42.23 \pm \\
4.99 ; \\
p>0.05 \\
\text { Stability index in } \\
\text { head straight EO } \\
\text { while standing on a } \\
\text { solid surface: } \\
\text { Pre: } 27.37 \pm 12.52 ; \\
\text { Post: } 28.40 \pm 15.11 ; \\
\text { P }<0.05\end{array}$ & $\begin{array}{l}\text { Weight bearing on } \\
\text { affected leg with head } \\
\text { straight EO while } \\
\text { standing on a solid } \\
\text { surface, } \% \text { : } \\
\text { Pre: } 40.81 \pm 10.12 ; \\
\text { Post: } 46.07 \pm 6.39 ; \\
p<0.05 \\
\text { Follow-up: } 46.72 \pm \\
8.80 ; \\
p>0.05 \\
\text { Weight bearing on } \\
\text { affected leg with head } \\
\text { straight EC while } \\
\text { standing on a solid } \\
\text { surface, } \%: \\
\text { Pre: } 41.10 \pm 9.86 ; \\
\text { Post: } 46.17 \pm 6.95 ; \\
\text { P }<0.05 \\
\text { Follow-up: } 45.86 \pm \\
7.98 ; \\
p>0.05 \\
\text { Stability index in } \\
\text { head straight EO } \\
\text { while standing on a } \\
\text { solid surface: } \\
\text { Pre: } 24.22 \pm 9.81 ; \\
\text { Post: } 25.69 \pm 8.92 ; \\
P<0.05\end{array}$ & $\begin{array}{c}\text { Statistically } \\
\text { significant } \\
\text { improvements in both } \\
\text { groups after } \\
\text { intervention in the } \\
\text { variables recorded by } \\
\text { posturography, FRT, } \\
\text { FES-I and TUG ( } p< \\
\text { 0.05). These results } \\
\text { were maintained at } \\
\text { 3-month follow-up in } \\
\text { FRT and TUG but not } \\
\text { in the variables } \\
\text { recorded by } \\
\text { posturography and } \\
\text { FES-I. } \\
\text { Not significant } \\
\text { statistical differences } \\
\text { between groups in } \\
\text { any variable after } \\
\text { intervention. }\end{array}$ & NR & $\begin{array}{c}\text { Intervention effect } \\
\text { Percentage of weight } \\
\text { bearing on affected } \\
\text { leg with head straight } \\
\text { EO while standing on } \\
\text { a solid surface: } \\
\text { 3.61/0.07 } \\
\text { Percentage of weight } \\
\text { bearing on affected } \\
\text { leg with head straight } \\
\text { EC while standing on } \\
\text { a solid surface: } \\
2.61 / 0.12 \\
\text { Stability index in } \\
\text { head straight EO } \\
\text { while standing on a } \\
\text { solid surface: } \\
0.02 / 0.89 \\
\text { Stability index in } \\
\text { head straight EC } \\
\text { while standing on a } \\
\text { solid surface: } \\
1.71 / 0.20 \text { FRT: } \\
0.00 / 0.96 \text { TUG: } \\
0.57 / 0.46 \text { FES-I: } \\
0.00 / 0.97\end{array}$ \\
\hline
\end{tabular}


Table 4. Cont

\begin{tabular}{|c|c|c|c|c|c|c|}
\hline Study & Outcomes & $\begin{array}{l}\text { Mean Values EG } \\
\quad(\text { Mean } \pm \text { SD })\end{array}$ & $\begin{array}{l}\text { Mean Values CG } \\
\quad(\text { Mean } \pm \text { SD) }\end{array}$ & Main Results & $\begin{array}{l}\text { Effect Size } \\
\text { (Cohen's d) }\end{array}$ & $\begin{array}{c}\text { Time } \times \text { Group } \\
\text { Interaction } \\
(\mathrm{F} / \mathrm{P})\end{array}$ \\
\hline & & $\begin{array}{l}\text { Follow-up: } 28.84 \pm \\
10.25 ; \\
p>0.05 \\
\text { Stability index in } \\
\text { head straight EC } \\
\text { while standing on a } \\
\text { solid surface: } \\
\text { Pre: } 43.34 \pm 27.73 ; \\
\text { Post: } 38.30 \pm 17.82 ; \\
\text { P }<0.05 \\
\text { Follow-up: } 38.52 \pm \\
\text { 23.46; } \\
p>0.05 \\
\text { FRT, cm: } \\
\text { Pre: } 21.62 \pm 5.19 ; \\
\text { Post: } 25.35 \pm 3.92 ; \\
p<0.05 \\
\text { Follow-up: } 24.63 \pm \\
4.82 ; \\
p<0.05 \\
\text { TUG, s: } \\
\text { Pre: } 26.06 \pm 12.05 ; \\
\text { Post: } 20.88 \pm 7.77 ; \\
p<0.05 \\
\text { Follow-up: } 23.01 \pm \\
7.89 ; \\
p<0.05 \\
\text { FES-I: } \\
\text { Pre: } 29.15 \pm 6.85 ; \\
\text { Post: } 25.38 \pm 6.06 ; \\
\text { P < } 0.05 \\
\text { Follow-up: } 29.31 \pm \\
9.81 ; \\
p>0.05\end{array}$ & $\begin{array}{l}\text { Follow-up: } 27.17 \pm \\
12.37 ; \\
p>0.05 \\
\text { Stability index in } \\
\text { head straight EC } \\
\text { while standing on a } \\
\text { solid surface: } \\
\text { Pre: } 35.17 \pm 13.45 ; \\
\text { Post: } 38.29 \pm 18.94 ; \\
\text { P }<0.05 \\
\text { Follow-up: } 36.86 \pm \\
\text { 16.52; } \\
p>0.05 \\
\text { FRT, cm: } \\
\text { Pre: } 21.73 \pm 5.33 ; \\
\text { Post: } 25.37 \pm 4.99 ; \\
p<0.05 \\
\text { Follow-up: } 25.82 \pm \\
7.29 ; \\
p<0.05 \\
\text { TUG, s: } \\
\text { Pre: } 29.45 \pm 16.22 ; \\
\text { Post: } 26.61 \pm 12.92 ; \\
p<0.05 \\
\text { Follow-up: } 25.60 \pm \\
\text { 13.79; } \\
p<0.05 \\
\text { FES-I: } \\
\text { Pre: } 30.07 \pm 4.76 ; \\
\text { Post: } 26.20 \pm 6.80 ; \\
\text { P < } 0.05 \\
\text { Follow-up: } 30.80 \pm \\
11.42 ; \\
p>0.05\end{array}$ & & & $\begin{array}{l}\text { Maintenance effect } \\
\text { Percentage of weight } \\
\text { bearing on affected } \\
\text { leg with head straight } \\
\text { EO while standing on } \\
\text { a solid surface: } \\
5.06 / 0.03 \\
\text { Percentage of weight } \\
\text { bearing on affected } \\
\text { leg with head straight } \\
\text { EC while standing on } \\
\text { a solid surface: } \\
2.61 / 0.12 \\
\text { Stability index in } \\
\text { head straight EO } \\
\text { while standing on a } \\
\text { solid surface: } \\
0.18 / 0.67 \\
\text { Stability index in } \\
\text { head straight EC } \\
\text { while standing on a } \\
\text { solid surface: } \\
1.71 / 0.20 \\
\text { FRT: } 0.16 / 0.69 \\
\text { TUG: } 0.07 / 0.79 \\
\text { FES-I: } 0.02 / 0.89\end{array}$ \\
\hline
\end{tabular}


Table 4. Cont.

\begin{tabular}{|c|c|c|c|c|c|c|}
\hline Study & Outcomes & $\begin{array}{c}\text { Mean Values EG } \\
(\text { Mean } \pm \text { SD) }\end{array}$ & $\begin{array}{c}\text { Mean Values CG } \\
\quad(\text { Mean } \pm \text { SD) }\end{array}$ & Main Results & $\begin{array}{l}\text { Effect Size } \\
\text { (Cohen's d) }\end{array}$ & $\begin{array}{c}\text { Time } \times \text { Group } \\
\text { Interaction } \\
(\mathbf{F} / \mathbf{P})\end{array}$ \\
\hline Kannan et al. [30] & $\begin{array}{l}\text { Posturography } \\
\text { (MVL) } \\
\text { BBS } \\
\text { TUG } \\
6 \text { MWT }\end{array}$ & $\begin{array}{l}\text { Data in graph, } \\
\text { Not extractable }\end{array}$ & $\begin{array}{l}\text { Data in graph, } \\
\text { Not extractable }\end{array}$ & $\begin{array}{c}\text { Statistically } \\
\text { significant } \\
\text { improvements after } \\
\text { intervention in EG in } \\
\text { MVL ( } p=0.001) . \\
\text { Statistically } \\
\text { significant } \\
\text { improvements in both } \\
\text { groups after } \\
\text { intervention in BBS, } \\
\text { TUG and } 6 \text { MWT ( } p< \\
0.05 \text { ). } \\
\text { Not statistically } \\
\text { significant differences } \\
\text { between groups in } \\
\text { any variable after } \\
\text { intervention. }\end{array}$ & NR & $\begin{array}{l}\text { MVL: } 14.295 / 0.001 \\
\text { NR in the other } \\
\text { variables }\end{array}$ \\
\hline Karasu et al. [31] & $\begin{array}{l}\text { Posturography } \\
\text { (displacement of the } \\
\text { centre of pressure in } \\
\text { different sensorial } \\
\text { conditions) } \\
\text { BBS } \\
\text { FRT } \\
\text { SBI } \\
\text { PASS } \\
\text { TUG }\end{array}$ & $\begin{array}{l}\text { displacement ML EO, } \\
\mathrm{cm}: \\
\text { pre: } 3.0 \pm 1.3 ; \\
\text { post: } 1.8 \pm 0.5 ; \\
p<0.05 \\
\text { follow-up: } 1.7 \pm 0.5 ; \\
p: \text { NR } \\
\text { displacement ML EC, } \\
\mathrm{cm}: \\
\text { pre: } 3.5 \pm 1.4 ; \\
\text { post: } 2.4 \pm 0.7 ; \\
p<0.05 \\
\text { follow-up: } 2.2 \pm 0.7 ; \\
p: \text { NR } \\
\text { displacement AP EO, } \\
\mathrm{cm} \\
\text { pre: } 3.0 \pm 0.9 ; \\
\text { post: } 2.4 \pm 0.8 ; \\
p<0.05 \\
\text { follow-up: } 2.05 \pm 0.7 ; \\
p: \text { NR } \\
\text { displacement AP EC, } \\
\mathrm{cm}\end{array}$ & $\begin{array}{l}\text { displacement ML EO, } \\
\mathrm{cm}: \\
\text { pre: } 2.7 \pm 0.9 ; \\
\text { post: } 2.4 \pm 0.9 ; \\
p<0.05 \\
\text { follow-up: } 2.5 \pm 0.6 ; \\
p: \text { NR } \\
\text { displacement ML EC, } \\
\mathrm{cm}: \\
\text { pre: } 3.1 \pm 1.1 ; \\
\text { post: } 2.9 \pm 1.3 ; \\
p<0.05 \\
\text { follow-up: } 3.1 \pm 0.6 ; \\
p: \text { NR } \\
\text { displacement AP EO, } \\
\mathrm{cm} \\
\text { pre: } 2.5 \pm 0.8 ; \\
\text { post: } 2.6 \pm 1.4 ; \\
p<0.05 \\
\text { follow-up: } 2.5 \pm 0.5 ; \\
p: \text { NR } \\
\text { displacement AP EC, } \\
\mathrm{cm}\end{array}$ & $\begin{array}{c}\text { Statistically } \\
\text { significant } \\
\text { improvements in both } \\
\text { groups after } \\
\text { intervention in } \\
\text { variables recorded by } \\
\text { posturography, BBS, } \\
\text { FRT, SBI and PASS ( } p \\
<0.05) \text {. } \\
\text { The improvements in } \\
\text { BBS and FRT were } \\
\text { statistical significantly } \\
\text { higher in the EG ( } p< \\
0.001) \text {. } \\
\text { TUG presented no } \\
\text { significant statistical } \\
\text { differences in any } \\
\text { group after } \\
\text { intervention }(p> \\
0.05) .\end{array}$ & NR & $\begin{array}{c}\text { Intervention effect } \\
\text { Displacement ML EO: } \\
\text { NR/0.034 } \\
\text { displacement ML EC: } \\
\text { NR/0.071 } \\
\text { displacement AP EO: } \\
\text { NR/0.012 } \\
\text { displacement AP EC: } \\
\text { NR/0.018 } \\
\text { BBS: NR/<0.001 } \\
\text { FRT: NR/ }<0.001 \\
\text { SBI: NR/0.068 } \\
\text { PASS: } \\
\text { NR/0.375 } \\
\text { TUG: NR/0.078 } \\
\text { Maintenance effect } \\
\text { NR }\end{array}$ \\
\hline
\end{tabular}


Table 4. Cont.

\begin{tabular}{|c|c|c|c|c|c|c|}
\hline Study & Outcomes & $\begin{array}{c}\text { Mean Values EG } \\
(\text { Mean } \pm \text { SD) }\end{array}$ & $\begin{array}{c}\text { Mean Values CG } \\
(\text { Mean } \pm \text { SD) }\end{array}$ & Main Results & $\begin{array}{l}\text { Effect Size } \\
\text { (Cohen's d) }\end{array}$ & $\begin{array}{c}\text { Time } \times \text { Group } \\
\text { Interaction } \\
(\mathrm{F} / \mathrm{P})\end{array}$ \\
\hline & & $\begin{array}{l}\text { pre: } 3.0 \pm 0.9 ; \\
\text { post: } 2.4 \pm 0.8 ; \\
p<0.05 \\
\text { follow-up: } 2.05 \pm 0.7 ; \\
p: \text { NR } \\
\text { displacement AP EC, } \\
\text { cm } \\
\text { pre: } 3.4 \pm 0.9 ; \\
\text { post: } 2.8 \pm 0.8 ; \\
p<0.05 \\
\text { follow-up: } 2.4 \pm 0.6 ; \\
p: \text { NR } \\
\text { BBS, score: } \\
\text { pre: } 38.8 \pm 6.9 ; \\
\text { post: } 48.9 \pm 6.4 ; \\
p<0.05 \\
\text { follow-up: } 48.7 \pm 4.7 ; \\
p: \text { NR } \\
\text { FRT, cm: } \\
\text { pre: } 16.4 \pm 5.5 ; \\
\text { post: } 25.2 \pm 5.5 ; \\
p<0.05 \\
\text { follow-up: } 23.6 \pm 5.4 ; \\
p: \text { NR } \\
\text { SBI, score: } \\
\text { pre: } 426.2 \pm 285.3 ; \\
\text { post: } 369.3 \pm 301.5 ; \\
p<0.05 \\
\text { follow-up: } 337 \pm \\
282.8 ; \\
p: \text { NR } \\
\text { PASS, score: } \\
\text { pre: } 28.8 \pm 4.3 ; \\
\text { post: } 32.5 \pm 2.5 ; \\
p<0.05 \\
\text { follow-up: } 32 \pm 2.4 ; \\
p: \text { NR } \\
\text { TUG, s: } \\
\text { pre: } 32.5 \pm 21.2 ; \\
\text { post: } 19.5 \pm 9.8 ; \\
p>0.05 \\
\text { follow-up: } 20.5 \pm 8.3 ; \\
p: \text { NR }\end{array}$ & $\begin{array}{l}\text { pre: } 2.5 \pm 0.8 ; \\
\text { post: } 2.6 \pm 1.4 ; \\
p<0.05 \\
\text { follow-up: } 2.5 \pm 0.5 ; \\
p \text { : NR } \\
\text { displacement AP EC, } \\
\text { cm } \\
\text { pre: } 3.1 \pm 0.7 ; \\
\text { post: } 3.2 \pm 1.4 ; \\
p<0.05 \\
\text { follow-up: } 3.3 \pm 0.6 ; \\
p: \text { NR } \\
\text { BBS, score: } \\
\text { pre: } 39.1 \pm 6.9 ; \\
\text { post: } 42.2 \pm 6.4 ; \\
p<0.05 \\
\text { follow-up: } 39.4 \pm 5.7 ; \\
p: \text { NR } \\
\text { FRT, cm: } \\
\text { pre: } 18.8 \pm 3.3 ; \\
\text { post: } 22.2 \pm 5.1 ; \\
p<0.05 \\
\text { follow-up: } 20 \pm 3.14) \\
p: \text { NR } \\
\text { SBI, score: } \\
\text { pre: } 412.4 \pm 196.8 ; \\
\text { post: } 314.2 \pm 129.8 ; \\
p<0.05 \\
\text { follow-up: } 399.7 \pm \\
74.7 ; \\
p: \text { NR } \\
\text { PASS, score: } \\
\text { pre: } 27.9 \pm 5.2 ; \\
\text { post: } 30.4 \pm 4.1 ; \\
p<0.05 \\
\text { follow-up: } 29.2 \pm 3.5 ; \\
p: \text { NR } \\
\text { TUG, s: } \\
\text { pre: } 27.4 \pm 15.0 ; \\
\text { post: } 24 \pm 13.5 ; \\
p>0.05 \\
\text { follow-up: } 29.6 \pm 10.5 ; \\
p: \text { NR }\end{array}$ & & & \\
\hline
\end{tabular}


Table 4. Cont.

\begin{tabular}{|c|c|c|c|c|c|c|}
\hline Study & Outcomes & $\begin{array}{l}\text { Mean Values EG } \\
(\text { Mean } \pm \text { SD) }\end{array}$ & $\begin{array}{l}\text { Mean Values CG } \\
(\text { Mean } \pm \text { SD) }\end{array}$ & Main Results & $\begin{array}{l}\text { Effect Size } \\
\text { (Cohen's d) }\end{array}$ & $\begin{array}{l}\text { Time } \times \text { Group } \\
\text { Interaction } \\
(\mathrm{F} / \mathrm{P})\end{array}$ \\
\hline Kim et al. [32] & $\begin{array}{l}\text { PASS } \\
\text { MMAS }\end{array}$ & $\begin{array}{l}\text { PASS, score: } \\
\text { Pre: } 25.8 \pm 3.85 \\
\text { Post: } 30.3 \pm 3.19 \\
p<0.05 \\
\text { MMAS, score: } \\
\text { Pre: } 29.8 \pm 6.4 \\
\text { Post: } 34.7 \pm 6.2 \\
p<0.05\end{array}$ & $\begin{array}{l}\text { PASS, score: } \\
\text { Pre: } 26.42 \pm 1.98 ; \\
\text { Post: } 28.0 \pm 1.63 ; \\
p<0.05 \\
\text { MMAS, score: } \\
\text { Pre: } 31.71 \pm 1.88 ; \\
\text { Post: } 33.57 \pm 1.51 \text {; } \\
p<0.05\end{array}$ & $\begin{array}{c}\text { Statistically } \\
\text { significant } \\
\text { improvements in both } \\
\text { groups after } \\
\text { intervention in PASS } \\
\text { and MMAS }(p<0.05) \text {. } \\
\text { These improvements } \\
\text { were statistical } \\
\text { significantly higher in } \\
\text { the EG }(p<0.05) .\end{array}$ & NR & NR \\
\hline Lee et al. [33] & $\begin{array}{l}\text { Wii Balance Board }{ }^{\circledR} \\
\text { (COP path length and } \\
\text { velocity in different } \\
\text { sensorial conditions) } \\
\text { FRT }\end{array}$ & $\begin{array}{l}\text { COP path length EO, } \\
\text { cm: } \\
\text { Pre: } 63.86 \pm 33.53 ; \\
\text { Post: } 51.25 \pm 27.29 ; \\
p<0.05 \\
\text { COP path length EC, } \\
\text { cm: } \\
\text { Pre: } 75.71 \pm 37.61 ; \\
\text { Post: } 75.71 \pm 37.61 ; \\
p<0.001 \\
\text { COP velocity EO, } \\
\text { cm/s: } \\
\text { Pre: } 2.12 \pm 1.11 ; \\
\text { Post: } 1.70 \pm 0.90 ; \\
p<0.05 \\
\text { COP velocity EC, } \\
\text { cm/s: } \\
\text { Pre: } 2.52 \pm 1.25 ; \\
\text { Post: } 1.99 \pm 0.79 ; \\
p<0.05 \\
\text { FRT, cm: } \\
\text { Pre: } 15.84 \pm 6.32 ; \\
\text { Post: } 24.75 \pm 7.44 ; \\
p<0.0001\end{array}$ & $\begin{array}{l}\text { COP path length EO, } \\
\text { cm: } \\
\text { Pre: } 60.65 \pm 30.14 ; \\
\text { Post: } 49.52 \pm 18.50 ; \\
p<0.05 \\
\text { COP path length EC, } \\
\text { cm: } \\
\text { Pre: } 65.85 \pm 34.95 ; \\
\text { Post: } 59.00 \pm 24.88 ; \\
p>0.05 \\
\text { COP velocity EO, } \\
\text { cm/s: } \\
\text { Pre: } 2.02 \pm 1.00 ; \\
\text { Post: } 1.65 \pm 0.61 ; \\
p<0.05 \\
\text { COP velocity EC, } \\
\text { cm/s: } \\
\text { Pre: } 2.19 \pm 1.16 ; \\
\text { Post: } 1.96 \pm 0.82 ; \\
p>0.05 \\
\text { FRT, cm: } \\
\text { Pre: } 16.40 \pm 5.91 ; \\
\text { Post: } 21.39 \pm 6.31 ; \\
p<0.0001\end{array}$ & $\begin{array}{c}\text { Statistically } \\
\text { significant } \\
\text { improvements in both } \\
\text { groups after } \\
\text { intervention in } \\
\text { variables recorded by } \\
\text { Wii Balance Board } \\
\text { and FRT }(p<0.05) \text {. } \\
\text { The improvements in } \\
\text { FRT were statistical } \\
\text { significantly higher in } \\
\text { the EG }(p<0.0001), \\
\text { but not in variables } \\
\text { recorded by Wii } \\
\text { Balance Board }{ }^{\circledR}(p> \\
0.05) .\end{array}$ & NR & NR \\
\hline
\end{tabular}


Table 4. Cont.

\begin{tabular}{|c|c|c|c|c|c|c|}
\hline Study & Outcomes & $\begin{array}{l}\text { Mean Values EG } \\
\quad(\text { Mean } \pm \text { SD })\end{array}$ & $\begin{array}{c}\text { Mean Values CG } \\
(\text { Mean } \pm \text { SD) }\end{array}$ & Main Results & $\begin{array}{l}\text { Effect Size } \\
\text { (Cohen's d) }\end{array}$ & $\begin{array}{c}\text { Time } \times \text { Group } \\
\text { Interaction } \\
(\mathbf{F} / \mathbf{P})\end{array}$ \\
\hline Lee et al. [34] & $\begin{array}{l}\text { BBS } \\
\text { FRT } \\
\text { TUG-cog }\end{array}$ & $\begin{array}{l}\text { BBS, score: } \\
\text { Pre: } 43.35 \pm 6.23 ; \\
\text { Post: } 46.19 \pm 5.57 ; \\
\text { P < } 0.05 \\
\text { Follow-up: } 46.31 \pm \\
5.80 ; \\
p<0.05 \\
\text { FRT, cm: } \\
\text { Pre: } 21.43 \pm 7.62 ; \\
\text { Post: } 22.63 \pm 5.07 ; \\
\text { P > } 0.05 \\
\text { Follow-up: } 22.48 \pm \\
\text { 5.87; } \\
p>0.05 \\
\text { TUG-cog, s: } \\
\text { Pre: } 27.18 \pm 14.90 ; \\
\text { Post: } 24.15 \pm 10.87 ; \\
p<0.05 \\
\text { Follow-up: } 23.52 \pm \\
\text { 10.96; } \\
p<0.05\end{array}$ & $\begin{array}{l}\text { BBS, score: } \\
\text { Pre: } 43.48 \pm 6.62 ; \\
\text { Post: } 45.71 \pm 6.64 ; \\
p<0.05 \\
\text { Follow-up: } 45.00 \pm \\
5.06 ; \\
p<0.05 \\
\text { FRT, cm: } \\
\text { Pre: } 22.05 \pm 8.27 ; \\
\text { Post: } 21.84 \pm 7.46 \text {; } \\
p>0.05 \\
\text { Follow-up: } 18.74 \pm \\
\text { 5.88; } \\
p>0.05 \\
\text { TUG-cog, s: } \\
\text { Pre: } 32.13 \pm 24.63 ; \\
\text { Post: } 28.48 \pm 21.53 ; \\
p<0.05 \\
\text { Follow-up: } 28.67 \pm \\
\text { 18.73; } \\
p<0.05\end{array}$ & $\begin{array}{l}\text { Statistically } \\
\text { significant } \\
\text { improvements in both } \\
\text { groups after } \\
\text { intervention and } \\
\text { follow-up in BBS and } \\
\text { TUG-cog }(p<0.05) \text {, } \\
\text { although without } \\
\text { significant differences } \\
\text { between both groups. } \\
\text { FRT presented no } \\
\text { significant statistical } \\
\text { differences in any } \\
\text { group after } \\
\text { intervention and } \\
\text { follow up }(p>0.05) .\end{array}$ & NR & $\begin{array}{c}\text { Intervention effect } \\
\text { BBS: 0.705/0.497 } \\
\text { FRT: 3.066/0.051 } \\
\text { TUG-cog: NR } \\
\text { Maintenance effect } \\
\text { NR }\end{array}$ \\
\hline Lee et al. [35] & $\begin{array}{l}\text { BBS } \\
\text { FRT } \\
\text { TUG }\end{array}$ & $\begin{array}{l}\text { BBS, score: } \\
\text { Pre: } 41.8 \pm 4.2 ; \\
\text { Post: } 46.2 \pm 4.3 ; \\
p:<0.05 \\
\text { FRT, cm: } \\
\text { Pre: } 20.4 \pm 3.5 ; \\
\text { Post: } 22.4 \pm 3.9 ; \\
p:<0.05 \\
\text { TUG, s: } \\
\text { Pre: } 16.6 \pm 4.3 ; \\
\text { Post: } 15.1 \pm 4.0 \text {; } \\
p:<0.05\end{array}$ & $\begin{array}{l}\text { BBS, score: } \\
\text { Pre: } 38.8 \pm 3.7 ; \\
\text { Post: } 41.2 \pm 2.9 ; \\
p:<0.05 \\
\text { FRT, cm: } \\
\text { Pre: } 17.8 \pm 0.9 ; \\
\text { Post: } 18.7 \pm 0.9 ; \\
p:<0.05 \\
\text { TUG, s: } \\
\text { Pre: } 18.1 \pm 2.7 \\
\text { Post: } 18.2 \pm 1.5 ; \\
p:>0.05\end{array}$ & $\begin{array}{c}\text { Statistically } \\
\text { significant } \\
\text { improvements in both } \\
\text { groups after } \\
\text { intervention in BBS } \\
\text { and FRT ( } p<0.05) \text {. } \\
\text { These improvements } \\
\text { were significantly } \\
\text { higher in the EG ( } p< \\
0.05) . \\
\text { Statistically } \\
\text { significant } \\
\text { improvements after } \\
\text { intervention in EG in } \\
\text { TUG ( } p<0.05) \text {. } \\
\text { These improvements } \\
\text { were statistically } \\
\text { significant between } \\
\text { both groups after } \\
\text { intervention }(p<0.05)\end{array}$ & NR & NR \\
\hline
\end{tabular}


Table 4. Cont.

\begin{tabular}{|c|c|c|c|c|c|c|}
\hline Study & Outcomes & $\begin{array}{l}\text { Mean Values EG } \\
\quad(\text { Mean } \pm \text { SD })\end{array}$ & $\begin{array}{l}\text { Mean Values CG } \\
\quad(\text { Mean } \pm \text { SD })\end{array}$ & Main Results & $\begin{array}{l}\text { Effect Size } \\
\text { (Cohen's d) }\end{array}$ & $\begin{array}{c}\text { Time } \times \text { Group } \\
\text { Interaction } \\
(\mathrm{F} / \mathrm{P})\end{array}$ \\
\hline Morone et al. [36] & $\begin{array}{l}\text { BBS } \\
\text { FAC } \\
10 \text { MWT }\end{array}$ & $\begin{array}{l}\text { Data in graph, } \\
\text { Not extractable }\end{array}$ & $\begin{array}{l}\text { Data in graph, } \\
\text { Not extractable }\end{array}$ & $\begin{array}{c}\text { Statistically } \\
\text { significant } \\
\text { improvements in both } \\
\text { groups after } \\
\text { intervention in all } \\
\text { variables (BBS, } \\
\text { FAC and 10 MWT) ( } p \\
<0.001), \\
\text { the improvements in } \\
\text { BBS and 10 MWT } \\
\text { were statistically } \\
\text { significantly higher in } \\
\text { the EG ( } p<0.05), \\
\text { but not in FAC ( } p> \\
0.05) . \\
\text { These Statistically } \\
\text { significant } \\
\text { improvements were } \\
\text { maintained in } \\
\text { follow-up }\end{array}$ & NR & $\begin{array}{c}\text { Intervention effect } \\
10 \text { MWT: NR/0.099 } \\
\text { NR in the other } \\
\text { variables } \\
\text { Maintenance effect } \\
\text { NR }\end{array}$ \\
\hline Park et al. [37] & $\begin{array}{l}\text { BBS } \\
\text { TUG } \\
10 \mathrm{MWT}\end{array}$ & $\begin{array}{l}\text { BBS, score: } \\
\text { Pre: } 35.80 \pm 8.61 ; \\
\text { Post: } 50.00 \pm 6.27 \\
p:<0.05 \\
\text { TUG, s: } \\
\text { Pre: } 44.35 \pm 33.49 ; \\
\text { Post: } 44.93 \pm 27.41 \text {; } \\
p:<0.05 \\
10 \text { MWT, m/s: } \\
\text { Pre: } 57.14 \pm 22.61 ; \\
\text { Post: } 44.73 \pm 20.87 ; \\
p:<0.05\end{array}$ & $\begin{array}{l}\text { BBS, score: } \\
\text { Pre: } 37.30 \pm 11.98 ; \\
\text { Post: } 44.70 \pm 7.47 ; \\
p:<0.05 \\
\text { TUG, s: } \\
\text { Pre: } 44.93 \pm 27.41 ; \\
\text { Post: } 41.16 \pm 27.68 \text {; } \\
p:<0.05 \\
10 \text { MWT, m/s: } \\
\text { Pre: } 53.90 \pm 26.06 ; \\
\text { Post: } 47.77 \pm 22.98 \\
p:<0.05\end{array}$ & $\begin{array}{c}\text { Statistically } \\
\text { significant } \\
\text { improvements in both } \\
\text { groups after } \\
\text { intervention in all } \\
\text { variables (BBS, } \\
\text { TUG and 10 MWT) ( } p \\
<0.05) \text { and } \\
\text { statistically } \\
\text { significantly higher in } \\
\text { the EG }(p<0.05)\end{array}$ & NR & NR \\
\hline
\end{tabular}


Table 4. Cont.

\begin{tabular}{|c|c|c|c|c|c|c|}
\hline Study & Outcomes & $\begin{array}{l}\text { Mean Values EG } \\
(\text { Mean } \pm \text { SD) }\end{array}$ & $\begin{array}{l}\text { Mean Values CG } \\
(\text { Mean } \pm \text { SD) }\end{array}$ & Main Results & $\begin{array}{l}\text { Effect Size } \\
\text { (Cohen's d) }\end{array}$ & $\begin{array}{c}\text { Time } \times \text { Group } \\
\text { Interaction } \\
(\mathrm{F} / \mathrm{P})\end{array}$ \\
\hline $\begin{array}{c}\text { Pedreira da Fonseca } \\
\text { et al. [38] }\end{array}$ & DGI & $\begin{array}{l}\text { Data in graph, } \\
\text { Not extractable }\end{array}$ & $\begin{array}{l}\text { Data in graph, } \\
\text { Not extractable }\end{array}$ & $\begin{array}{c}\text { Statistically } \\
\text { significant } \\
\text { improvements in CG } \\
\text { after intervention in } \\
\text { DGI }(p<0.05) \text {. } \\
\text { Clinical } \\
\text { improvements in EG } \\
\text { after intervention in } \\
\text { DGI, but not } \\
\text { statistically significant } \\
\quad(p>0.05)\end{array}$ & NR & NR \\
\hline Rajaratnam et al. [39] & $\begin{array}{l}\text { Wii Balance Board }{ }^{\circledR} \\
\text { (COP) } \\
\text { BBS } \\
\text { FRT } \\
\text { TUG }\end{array}$ & Data not extractable & Data not extractable & $\begin{array}{c}\text { Statistically } \\
\text { significant } \\
\text { improvements after } \\
\text { intervention in EG in } \\
\text { FRT and TUG ( } p< \\
\text { 0.05) and in CG in } \\
\text { TUG ( } p<0.05) \text {. } \\
\text { Variable recorded by } \\
\text { Wii Balance Board }{ }^{\circledR} \\
\text { presented no } \\
\text { significant statistical } \\
\text { differences in any } \\
\text { group after } \\
\text { intervention }(p>0.05) . \\
\text { No significant } \\
\text { statistical differences } \\
\text { between both groups } \\
\text { in any variables after } \\
\text { intervention }(p>0.05)\end{array}$ & NR & NR \\
\hline
\end{tabular}


Table 4. Cont.

\begin{tabular}{|c|c|c|c|c|c|c|}
\hline Study & Outcomes & $\begin{array}{c}\text { Mean Values EG } \\
(\text { Mean } \pm \text { SD) }\end{array}$ & $\begin{array}{l}\text { Mean Values CG } \\
\quad(\text { Mean } \pm \text { SD })\end{array}$ & Main Results & $\begin{array}{l}\text { Effect Size } \\
\text { (Cohen's d) }\end{array}$ & $\begin{array}{c}\text { Time } \times \text { Group } \\
\text { Interaction } \\
\text { (F/P) }\end{array}$ \\
\hline Singh et al. [40] & $\begin{array}{l}\text { Posturography } \\
\text { (OBS) } \\
\text { TUG } \\
10 \mathrm{MWT} \\
6 \mathrm{MWT}\end{array}$ & $\begin{array}{l}\text { OBS, score: } \\
\text { Pre: } 2.53 \pm 1.02 ; \\
\text { Post: } 2.70 \pm 0.72 ; \\
p:>0.05 \\
\text { TUG, s: } \\
\text { Pre: } 25.33 \pm 14.38 ; \\
\text { Post: } 23.07 \pm 12.22 \text {; } \\
p:>0.05 \\
10 \text { MWT, m/s: } \\
\text { Pre: } 13.20 \pm 7.46 ; \\
\text { Post: } 12.17 \pm 6.16 ; \\
p:>0.05 \\
6 \text { MWT, m: } \\
\text { Pre: } 162.40 \pm 78.97 ; \\
\text { Post: } 165.27 \pm 78.52 ; \\
p:>0.05\end{array}$ & $\begin{array}{l}\text { OBS, score: } \\
\text { Pre: } 3.25 \pm 1.12 ; \\
\text { Post: } 3.31 \pm 1.39 ; \\
p:>0.05 \\
\text { TUG, s: } \\
\text { Pre: } 23.27 \pm 12.15 ; \\
\text { Post: } 21.69 \pm 12.29 ; \\
p:>0.05 \\
10 \text { MWT, m/s: } \\
\text { Pre: } 14.80 \pm 10.16 ; \\
\text { Post: } 15.03 \pm 9.35 ; \\
p:>0.05 \\
\text { 6 MWT, m: } \\
\text { Pre: } 209.92 \pm 176.53 ; \\
\text { Post: } 167.23 \pm 103.85 ; \\
p:>0.05\end{array}$ & $\begin{array}{l}\text { No significant } \\
\text { statistical differences } \\
\text { were found in any } \\
\text { group after } \\
\text { intervention in any } \\
\text { variable }(p>0.05)\end{array}$ & NR & $\begin{array}{c}\text { TUG: NR/0.02 } \\
\text { 10 MWT: NR/0.66 } \\
6 \text { MWT: NR/0.59 } \\
\text { OBS: NR }\end{array}$ \\
\hline Song \& Park [41] & $\begin{array}{l}\text { Posturography } \\
\text { (WB on the affected } \\
\text { side, anterior and } \\
\text { posterior LOS) } \\
\text { TUG } \\
10 \text { MWT }\end{array}$ & $\begin{array}{l}\text { Affected side WB, } \% \text { : } \\
\text { Pre: } 42.8 \pm 6.1 ; \\
\text { Post: } 46.1 \pm 4.3 ; \\
p:<0.05 \\
\text { Forward LOS, mm: } \\
\text { Pre: } 2732.9 \pm 3137.1 ; \\
\text { Post: } 3311.7 \pm 3786.5 ; \\
p:<0.05 \\
\text { Backward LOS, mm: } \\
\text { Pre: } 2072.7 \pm 2050.4 ; \\
\text { Post: } 1895.9 \pm 2097.5 ; \\
p:<0.05 \\
\text { TUG, s: } \\
\text { Pre: } 21.17 \pm 7.7 ; \\
\text { Post: } 21.9 \pm 7.9 ; \\
p:<0.05 \\
10 \mathrm{MWT}, \mathrm{m} / \mathrm{s}: \\
\text { Pre: } 18.3 \pm 2.2 ; \\
\text { Post: } 21.4 \pm 8.9 ; \\
p:<0.05\end{array}$ & $\begin{array}{l}\text { Affected side WB, } \% \text { : } \\
\text { Pre: } 42.1 \pm 4.3 ; \\
\text { Post: } 43.3 \pm 4.9 ; \\
p:<0.05 \\
\text { Forward LOS, mm: } \\
\text { Pre: } 5670.8 \pm 4291.1 ; \\
\text { Post: } 4322.6 \pm 3565.5 ; \\
p:<0.05 \\
\text { Backward LOS, mm: } \\
\text { Pre: } 3971.7 \pm 2794.3 ; \\
\text { Post: } 2889.7 \pm 2769.7 ; \\
p:<0.05 \\
\text { TUG, s: } \\
\text { Pre:16.6 } \pm 4.7 ; \\
\text { Post: } 19.5 \pm 7.5 ; \\
p:<0.05 \\
10 \text { MWT, m/s: } \\
\text { Pre: } 14.3 \pm 2.1 ; \\
\text { Post: } 19.1 \pm 8.8 ; \\
p:<0.05\end{array}$ & $\begin{array}{c}\text { Thatistically } \\
\text { significant } \\
\text { improvements in both } \\
\text { groups after } \\
\text { intervention in all } \\
\text { variables (Variables } \\
\text { recorded by } \\
\text { posturography, } \\
\text { TUG and } 10 \text { MWT) ( } \\
<0.05) \text { and statically } \\
\text { significant higher in } \\
\text { the EG }(p<0.05)\end{array}$ & NR & NR \\
\hline
\end{tabular}


Table 4. Cont.

\begin{tabular}{|c|c|c|c|c|c|c|}
\hline Study & Outcomes & $\begin{array}{l}\text { Mean Values EG } \\
(\text { Mean } \pm \text { SD) }\end{array}$ & $\begin{array}{c}\text { Mean Values CG } \\
(\text { Mean } \pm \text { SD) }\end{array}$ & Main Results & $\begin{array}{l}\text { Effect Size } \\
\text { (Cohen's d) }\end{array}$ & $\begin{array}{c}\text { Time } \times \text { Group } \\
\text { Interaction } \\
(\mathrm{F} / \mathrm{P})\end{array}$ \\
\hline Yatar \& Yildrim [42] & $\begin{array}{l}\text { Wii Balance Board }{ }^{\circledR} \\
\text { (weight distribution) } \\
\text { FRT } \\
\text { BBS } \\
\text { TUG } \\
\text { DGI }\end{array}$ & $\begin{array}{l}\text { Weight distribution, } \\
\text { paretic side, } \%: \\
\text { Pre: } 44.04 \pm 7.47 ; \\
\text { Post: } 48.00 \pm 2.80 ; \\
p: \leq 0.05 \\
\text { Follow-up: } 46.64 \pm \\
4.40 ; \\
p:>0.05 \\
\text { FRT, cm: } \\
\text { Pre: } 25.26 \pm 5.92 ; \\
\text { Post: } 29.40 \pm 5.16 ; \\
p: \leq 0.05 \\
\text { Follow-up: } 30.06 \pm \\
\text { 5.16; } \\
p:>0.05 \\
\text { BBS, score: } \\
\text { Pre: } 45.60 \pm 5.26 ; \\
\text { Post: } 50.33 \pm 4.09 ; \\
\text { P: } \leq 0.05 \\
\text { Follow-up: } 50.33 \pm \\
4.16 ; \\
p:>0.05 \\
\text { TUG, s: } \\
\text { Pre: } 17.96 \pm 7.77 ; \\
\text { Post: } 16.17 \pm 8.23 ; \\
\text { P: } \leq 0.05 \\
\text { Follow-up: } 15.95 \pm \\
7.93 ; \\
p:>0.05 \\
\text { DGI, score: } \\
\text { Pre: } 14.86 \pm 4.12 ; \\
\text { Post: } 16.86 \pm 3.35 ; \\
\text { P: } \leq 0.05 \\
\text { Follow-up: } 16.86 \pm \\
3.33 ; \\
p:>0.05\end{array}$ & $\begin{array}{l}\text { Weight distribution, } \\
\text { paretic, } \%: \\
\text { Pre: } 47.54 \pm 10.16 ; \\
\text { Post: } 48.59 \pm 8.38 ; \\
\text { p: }>0.05 \\
\text { Follow-up: } 48.14 \pm \\
8.94 ; \\
p:>0.05 \\
\text { FRT, cm: } \\
\text { Pre: } 24.20 \pm 6.66 ; \\
\text { Post: } 27.13 \pm 6.19 ; \\
\text { p: } \leq 0.05 \\
\text { Follow-up: } 26.80 \pm \\
6.58 ; \\
p:>0.05 \\
\text { BBS, score: } \\
\text { Pre: } 39.60 \pm 9.31 ; \\
\text { Post: } 44.80 \pm 7.48 ; \\
\text { P: } \leq 0.05 \\
\text { Follow-up: } 44.20 \pm \\
7.77 ; \\
p: \leq 0.05 \\
\text { TUG, s: } \\
\text { Pre: } 26.36 \pm 11.60 ; \\
\text { Post: } 22.11 \pm 11.88 ; \\
\text { P: } \leq 0.05 \\
\text { Follow-up: } 22.73 \pm \\
11.95 ; \\
p:>0.05 \\
\text { DGI, score: } \\
\text { Pre: } 12.60 \pm 3.96 ; \\
\text { Post: } 14.46 \pm 3.48 ; \\
\text { P: } \leq 0.05 \\
\text { Follow-up: } 14.86 \pm \\
4.06 ; \\
p:>0.05\end{array}$ & 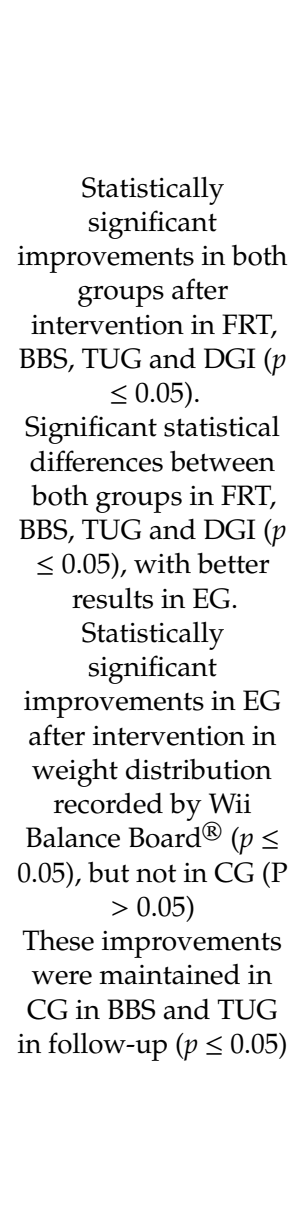 & NR & NR \\
\hline
\end{tabular}

10 MWT, 10-metre walk test; 6 MWT, 6-min walk test; AP, anteroposterior; BBS, Berg Balance Scale; COP, centre of pressure; DGI, Dynamic Gait Index; EC, eyes closed; EO, eyes open; FAC, functional outpatient category; FES-I, Falls Efficacy Scale International; FRT, Functional Reach Test; LOS, limit of stability; ML, mediolateral; MMAS, modified motor assessment scale; MVL, movement velocity; NR, not reported; OBS, Overall Balance Score; PASS, Postural Assessment Scale; post, values measured after intervention; pre, values measured before intervention; PSV, Postural Sway Velocity; SBI, Static Balance Index; SD, standard deviation TUG, Timed Up and Go test; TUG-cog, cognitive Timed Up and Go test and WB, weight bearing. 


\subsection{Quality of Evidence}

Nine studies showed moderate bias [26,27,30,32,33,36,40-42], and the other nine studies showed low bias $[25,28,29,31,34,35,37-39]$. Performance bias was the most common one. However, notification bias only appeared in one study [39] (Table 5 and Figure 2).

Table 5. Bias risk assessment of the articles included.

\begin{tabular}{|c|c|c|c|c|c|c|}
\hline \multirow[b]{2}{*}{ REFERENCES } & \multicolumn{6}{|c|}{ BIAS } \\
\hline & Selection & Performance & Detection & Wear & Notification & Others \\
\hline Barcala et al. [25] & + & - & + & + & + & + \\
\hline Bower et al. [26] & - & - & + & - & + & + \\
\hline Cho et al. [27] & + & - & - & + & + & + \\
\hline Hung et al. [28] & + & - & + & + & + & + \\
\hline Hung et al. [29] & + & - & + & + & + & + \\
\hline Kannan et al. [30] & + & - & - & + & + & + \\
\hline Karasu et al. [31] & + & - & + & + & + & + \\
\hline Kim et al. [32] & + & - & - & + & + & + \\
\hline Lee et al. [33] & - & - & - & + & + & + \\
\hline Lee et al. [34] & + & - & + & + & + & + \\
\hline Lee et al. [35] & + & - & + & + & + & + \\
\hline Morone et al. [36] & + & - & + & - & + & + \\
\hline Park et al. [37] & + & - & + & + & + & + \\
\hline $\begin{array}{c}\text { Pedreira da } \\
\text { Fonseca et al. [38] }\end{array}$ & + & - & + & + & + & + \\
\hline $\begin{array}{l}\text { Rajaratnam et al. } \\
{[39]}\end{array}$ & + & + & + & + & - & + \\
\hline Singh et al. [40] & - & - & + & + & + & + \\
\hline Song \& Park [41] & + & - & - & + & + & + \\
\hline $\begin{array}{c}\text { Yatar \& Yildrim } \\
{[42]}\end{array}$ & - & - & - & + & + & + \\
\hline
\end{tabular}

Coding: + = "no bias" and - = "presence of bias".

SELECTION BIAS

PERFORMANCE BIAS

DETECTION BIAS

WEAR BIAS

NOTIFICATION BIAS

OTHER BIAS

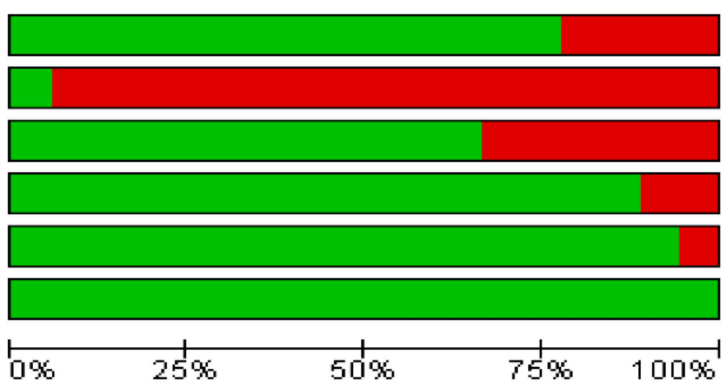

Low risk of bias

\section{Doubtful risk of bias}

\section{High risk of}

bias

Figure 2. Risk of bias graph. 
The lowest scores obtained on the PEDro scale were 4/10 [42] and 5/10 [40], and in the rest of the studies, the scores were between $6 / 10$ and 8/10 (Table 3).

\section{Discussion}

The aim of this paper was to investigate the effects of commercial video games as low-cost semi-immersive VR systems on balance and gait in stroke patients and to know the differences between the different devices and video games used. Previous publications have shown positive effects using immersive VR systems [14-16,44-46] on balance and gait in different neurological conditions. However, this equipment is costly and complex to use and maintain, unlike the low-cost video game systems [19] included in the papers contemplated in this SR.

As regards the studies included in this SR, the results generally show positive effects on balance and gait in post-stroke patients. Specifically, in relation to the participants, they possess homogeneous characteristics regarding age, gender and type of stroke. On the contrary, homogeneity is not observed in progression times, including participants in chronic and subacute states. However, differences have not been observed in the results as regards to patient age and progression times, contrary to what was described in previous studies as regards the benefits of physical therapy after stroke, which showed that the oldest patients obtained lower levels of improvement and, also, that patients with the most mild condition recuperated more favourably [47,48]. However, effect size and time $\mathrm{x}$ group interaction were included in almost none of the papers analysed in this SR.

The assessment tools used to quantify the effects of therapy on balance and gait were heterogeneous in the studies included, which evidences a lack of standardisation in the assessment protocols of balance and gait in post-stroke patients. In several papers, objective tools were used, such as computerised dynamic posturography systems, which are considered to be baseline instrumentation methods for assessing balance $[49,50]$ and, in other cases, observational functional assessment tests, such as TUG or BBS, which, although amply validated in this clinical context [51-55], knowing how to apply them depends on the examiner's experience. Moreover, in this last test, the floor ceiling effect is frequent, which means it is not sensitive to discreet changes in patients with mild or very severe balance disorders $[53,55]$.

Results from all the studies show improvements in some of the aspects analysed, regardless of the approach (with or without commercial video games) that is to say, performing rehabilitation leads to improvements in patients who have suffered a stroke, which is in-line with previous reviews [56-58]. However, specifically regarding balance, no changes occurred in several of the papers included in this SR $[27,39,40,42]$ or after conventional physiotherapy treatment or when the latter was applied in conjunction with exercises in a virtual environment. However, improvements in balance during functional tasks were observed. This could be due to the fact that such tasks allow greater adaptation as regards the support base, as well as the need to perform weight transfers in a more dynamic way, facilitating postural stability, whereas maintaining bipedestations in the tests used for assessment means the support base must be kept constant, as well as the load and support points when straightening up.

The results observed also seem to be linked to the length of the therapy sessions. In six studies, the session times were longer in the EG compared to the CG $[25,27,31,32,35,37]$. In one of the studies, this aspect did not lead to significant differences in the results obtained for both groups [25], in contrast with the other five studies, which showed differences between the groups (in favour of the EG) $[27,31,32,35,37]$. Thus, there does seem to be a relationship between the length of time of treatment and the results, as suggested in previous studies as regards the effects of physiotherapy treatment on strokes [48,59]. In terms of duration, the number of sessions and weekly frequency of rehabilitation programmes with commercial video games, there was variability between the studies included in this SR. Overall, the average number of sessions was nine in two studies [26,32] or 42 sessions in another one [37]. No differences were found in the results in relation to the total duration of the intervention weeks, the number of total sessions or the weekly frequency of the sessions. 
On the other hand, in the majority of the studies where no difference existed in the session times of treatment between the groups, significant statistical differences were found in balance and/or gait in favour of the EG $[33,36,39,41,42]$. Some authors suggested that these improvements in the EG were due to patients being highly motivated and thanks to the possibility offered by these systems to constantly adapt the activities to the demands of participants. Commercial video consoles offer accessible tasks, but they are challenging and have the possibility of training skills, which cannot be performed in a natural way in real life; this motivation encourages participation and concentration during therapy [17-19,41]. Likewise, these devices allow variable practice of repetitive tasks similar to those performed in their daily routine, which favours the efficient transfer of learning and, thus, enables the tasks to be performed in different contexts and situations, as indicated by several authors [60]. The feedback provided by these devices is also important, since auditory and visual signals inform of errors when performing the movements. Thus, the user is aware of his/her posture and movements at all times, activating brain circuits similar to those that would be activated with the same practice in real life [41,60]. Most of the studies show the main advantages (Table 6) related to the "active ingredients" that make VR interventions effective in promoting recovery in stroke patients. The therapeutic advantages are related to principles relevant to neuroplasticity, such as providing goal-oriented tasks, increasing repetition and dosage, providing therapists and patients with additional feedback and allowing to adjust the task difficulty. In addition, it has been suggested that the use of VR increases patient motivation, enjoyment, and engagement; makes intensive task-relevant training more interesting and offers enriched environments.

Table 6. Advantages and disadvantages of commercial video games.

\begin{tabular}{|c|c|}
\hline Advantages & Disadvantages \\
\hline $\begin{array}{ll}- & \text { High motivation } \\
- & \text { Increase therapeutic adherence } \\
- & \text { Low cost } \\
- & \text { Acquisition very accessible in stores } \\
- & \text { Easy to use } \\
- & \text { Easy interaction } \\
- & \text { Allow variable and repetitive practice } \\
- & \text { Provide goal-oriented tasks } \\
- & \text { Constant adaptation of video games (allowing } \\
- & \text { to adjust task difficulty) } \\
- & \text { controlled environment } \\
- & \text { Ludic and social activity } \\
- & \text { Possibility of using them at home } \\
- & \text { Possibility of using them autonomously, } \\
\text { without needing constant supervision from }\end{array}$ & 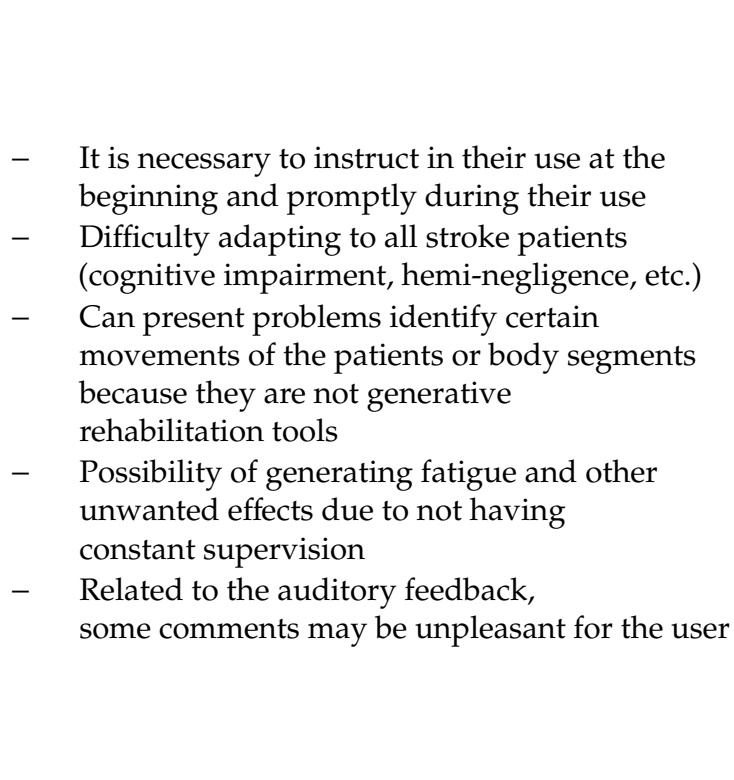 \\
\hline
\end{tabular}

The video consoles used were different as regards the ways to interact with the systems and the virtual environments. A remote control is needed in order to use the $\mathrm{Wii}^{\circledR}$ console, which functions through a Bluetooth connection. Due to their upper trunk pathology, this is not something that all patients affected by stroke can do, as is seen in one of the studies included in the SR [32]. Therefore, some patients need an adaptor to be able to hold the control or a system for holding it in their hand. Good balance in bipedestations is required in order to use the Wii Balance Board ${ }^{\circledR}$, as the individual must remain on the board in a restricted area; thus, his/her support base is reduced and at a height of approximately three $\mathrm{cm}$ from the floor. This limits the possibility of using it with a 
larger range of patients. When using the Kinect ${ }^{\circledR}$ by Xbox-Microsoft ${ }^{\circledR}$ device, the user acts as the remote control for the console, as it uses infrared technology that generates a virtual identity or avatar and simulates the individual's motion. Therefore, the use of controls is not required, and the game space is not limited, but rather, it forces constant change in the centre of mass in different directions in order to start and interact with the system [37,39]. However, the use of Kinect requires the sensor to identify all body segments, which makes it difficult to use in patients who need partial support for standing. Despite these differences, the majority of studies analysed used the $\mathrm{Wii}^{\circledR}{ }^{\circledR}$ with its balance board, thus implying that patients had good balance in bipedestations and were able to maintain their standing balance for several minutes. Indistinct uses of either of the devices ( $\mathrm{Wii}^{\circledR}$ or Kinect-Xbox ${ }^{\circledR}$ ) or a combined use did not show differences in the results obtained. Therefore, as to the results, they were not influenced by the fact that the games included displacements and load transfers, which is the main difference between video games that used the Wii Balance Board ${ }^{\circledR}$ and the rest of the video games in which player space was not limited.

It is important to evaluate the requirements of controller handling and game space in relation to the freedom of movement of patients, especially in relation to the new video consoles available on the market, because the video games used in rehabilitation so far and described in this SR are not available on the market by now, and new video consoles have emerged that will replace these. In this respect, there is a new semi-immersive system available on the market, Nintendo Switch ${ }^{\circledR}$, which (like the $\mathrm{Wii}^{\circledR}$ ) works with a controller with a Bluetooth connection (unadapted) but without the need for a platform. In this sense, systems based on infrared technology have made it possible to overcome the limitations observed in the handling of controllers and travel space of people with strokes. As happens with the video console PlayStation $5^{\circledR}$, which will be launched to the market (expected to at the end of 2020), it will include motion capture hardware similar to that in the $\mathrm{Xbox}^{\circledR}$ and its device $\mathrm{Kinec}^{\circledR}$. This console, with motion capture technology, is also compatible with VR glasses, as PlayStation ${ }^{\circledR}$ VR is now available on the market. Unlike the consoles included in this review, they are immersive VR systems. Additionally, the new Xbox series $X^{\circledR}$ will be launched to the market (expected at the end of 2020), but Microsoft ${ }^{\circledR}$ has not reported if it will be compatible with any VR support, as is the case with the Xbox One ${ }^{\circledR}$ (not compatible with the Kinect ${ }^{\circledR}$ device) available now for sale; even so, there are speculations about the possible creation of new video consoles after Microsoft ${ }^{\circledR}$ registered a patent at the end of 2019 related to VR in the Xbox ${ }^{\circledR}$, named Xbox VR Mat ${ }^{\circledR}$, in which the movement of the player is controlled by a peripheral device similar to Kinect ${ }^{\circledR}$. The sensory tolerance, ease of interaction and understanding of the tasks of semi-immersive systems have been key aspects of their use as a complement to balance training programmes in patients with strokes, so it would be necessary to study and compare the ability of interactions of these patients with fully immersive technologies to assess the suitability of use of the new generation immersive consoles in the field of motor rehabilitation.

With respect to the video games used, it is important to consider the exercises offered by each of them rather than the video game; they should be adapted to the functional state of the patient. They should begin with games that involve small displacements of the centre of mass, and as the postural control improves, they must evolve greater displacements at higher speeds and should include more complex actions, such unipodal postures or jumps. In some studies, these devices are considered as an option to free up time for the therapist $[39,41]$. In our clinical experience, the integration of video game systems into stroke rehabilitation programmes should be understood as an opportunity to enrich the patient's sensory experience in relation to balance, as well as an element of therapeutic motivation and adherence.

Regarding the methodological quality of the included papers in this SR, the most common bias was performance bias, which was in all studies, except for one [39]. This risk of bias was produced by the lack of blinding of participants or therapists. The same thing happened in PEDro, as the decrease in PEDro scores was mainly due to the lack of blinding of participants or therapists.

Although an extensive bibliographic search was carried out and the methods for selecting and extracting data were thorough, publication bias cannot be discarded, as only studies published in 
journals were retrieved under our inclusion and exclusion criteria. Effect size and time $\mathrm{x}$ group interactions were included in almost none of the papers analysed in this SR. Additionally, our results should not be extrapolated to other neurological disorders and other outcome measures. An ideal protocol of treatment with video consoles and video games for balance and gait rehabilitation in stroke patients cannot be offered based on this SR, because the results of this SR did not indicate the most effective time to use the intervention in recovery (acute, subacute or chronic stroke); the most effective video console or video game or the most effective dosage (session times, weekly frequencies and duration of interventions).

\section{Conclusions}

Commercial video game systems combined with conventional rehabilitation treatments have shown positive effects in treating balance and gait disorders in post-stroke patients. Video game consoles should be prescribed and supervised by a professional rehabilitation practitioner while post-stroke patients maintain their conventional treatments. Commercial video game consoles for balance and gait rehabilitation purposes must suit the functional state of the patient to carry out the necessary adaptations during the rehabilitation sessions. It is not clear which video console or video game presents the most evidence to treat balance and gait in stroke patients or in which stroke phase this intervention is better or the appropriate dosage to produce these improvements.

Supplementary Materials: The following are available online at http://www.mdpi.com/2076-3417/10/18/6426/s1: Table S1: Comprehensive description of the articles included in the systematic review.

Author Contributions: Conceptualization, S.P.-I., R.M.O.-G. and R.C.-d.-1.-C.; methodology, S.P.-I., R.M.O.-G. and R.C.-d.-1.-C.; writing - original draft preparation, S.P.-I., R.M.O.-G. and R.C.-d.-1.-C.; writing-review and editing, S.P.-I., R.M.O.-G. and R.C.-d.-1.-C. and supervision, R.M.O.-G. and R.C.-d.-1.-C. All authors have read and agreed to the published version of the manuscript.

Funding: This research received no external funding.

Acknowledgments: The authors would like to thank the Rey Juan Carlos University Library and Alberto Arribas and Arántzazu Nuñez Cascajero for their contributions.

Conflicts of Interest: The authors declare no conflict of interest.

\section{References}

1. WHO MONICA Project Principal Investigators. The World Health Organization MONICA Project (monitoring trends and determinants in cardiovascular disease): A major international collaboration. WHO MONICA Project Principal Investigators. J. Clin. Epidemiol. 1988, 41, 105-114. [CrossRef]

2. World Heart Organization Stroke. Neurological Disorders: Public Health Challenges; World Heart Organization Stroke: Geneva, Switzerland, 2006; pp. 151-163.

3. Federación Española de Ictus. Código Ictus. Available online: https://ictusfederacion.es/infoictus/codigoictus/ (accessed on 23 June 2020).

4. Ordahan, B.; Ay, K.; Basaran, A.; Turkoglu, G.; Kucuksarac, S.; Cubukcu, M.; Tekin, L.; Polat, A.D.; Kuran, B. Impact of exercises administered to stroke patients with balance trainer on reha-bilitation results: A randomized controlled study. Hippokratia 2015, 19, 125-130. [PubMed]

5. Latham, N.K.; Jette, D.U.; Slavin, M.; Richards, L.G.; Procino, A.; Smout, R.J.; Horn, S.D. Physical therapy during stroke rehabilitation for people with different walking abilities. Arch. Phys. Med. Rehabil. 2005, 86, 41-50. [CrossRef] [PubMed]

6. Weiss, P.L.; Kizony, R.; Feintuch, U.; Katz, N. Virtual reality in neurorehabilitation. In Textbook of Neural Repair and Rehabilitation; Selzer, M., Clarke, S., Cohen, L., Duncan, P., Gage, F., Eds.; Cambridge University Press: Cambridge, UK, 2010; pp. 182-197.

7. Olguín Carbajal, M.; Rivera Zárate, I.; Hernández Montañez, E. Introducción a la Realidad Virtual. Polibits 2006, 33, 11-15. [CrossRef]

8. Bayón, M.; Martínez, J. Rehabilitación del ictus mediante realidad virtual. Rehabilitacion 2010, 44, $256-260$. [CrossRef] 
9. Deutsch, J.E.; Lewis, J.A.; Burdea, G. Technical and patient performance using a virtual reality-integrated Telerehabilitation system: Preliminary finding. IEEE Trans. Neural Syst. Rehabil. Eng. 2007, 15, 30-35. [CrossRef]

10. Piron, L.; Turolla, A.; Agostini, M.; Zucconi, C.; Cortese, F.; Zampolini, M.; Zannini, M.; Dam, M.; Ventura, L.; Battauz, M.; et al. Exercises for paretic upper limb after stroke: A combined virtual-reality and telemedicine approach. J. Rehabil. Med. 2009, 41, 1016-1020. [CrossRef]

11. Grigoras, A.V.; Matei, D.; Ignat, E.B. Non-Immersive Virtual Reality for Upper Limb Rehabilitation in Stroke Survivors-A Feasibility Study. Balneo Res. J. 2018, 9, 232-239. [CrossRef]

12. Törnbom, K.; Danielsson, A. Experiences of treadmill walking with non-immersive virtual reality after stroke or acquired brain injury-A qualitative study. PLoS ONE 2018, 13, e0209214. [CrossRef]

13. Cortés-Pérez, I.; Nieto-Escamez, F.A.; Obrero-Gaitán, E. Immersive Virtual Reality in Stroke Patients as a New Approach for Reducing Postural Disabilities and Falls Risk: A Case Series. Brain Sci. 2020, 10, 296. [CrossRef]

14. Laver, K.; George, S.; Thomas, S.; Deutsch, J.; Crotty, M. Virtual reality for stroke rehabilitation (Review) summary of findings for the main comparison. Virtual Real. Stroke Rehabil. 2017, 80, 57-62.

15. Li, Z.; Han, X.-G.; Sheng, J.; Ma, S.J. Virtual reality for improving balance in patients after stroke: A systematic review and meta-analysis. Clin. Rehabil. 2015, 30, 432-440. [CrossRef]

16. Maier, M.; Rubio Ballester, B.; Duff, A.; Duarte Oller, E.; Verschure, P.F.M.J. Effect of Specific Over Nonspecific VR-Based Rehabilitation on Poststroke Motor Recovery: A Systematic Meta-analysis. Neurorehabil. Neural Repair 2019, 33, 112-129. [CrossRef] [PubMed]

17. Pérez-Castilla Alvarez, L. Buenas Prácticas de Accesibilidad en Videojuegos, 1st ed.; Ministerio de Sanidad, Servicios Sociales e Igualdad: Madrid, Spain, 2012.

18. Deutsch, J.E.; Borbely, M.; Filler, J.; Huhn, K.; Guarrera-Bowlby, P. Use of a Low-Cost, Commercially Available Gaming Console (Wii) for Rehabilitation of an Adolescent With Cerebral Palsy. Phys. Ther. 2008, 88, 1196-1207. [CrossRef] [PubMed]

19. Burke, J.W.; McNeill, M.D.J.; Charles, D.K.; Morrow, P.J.; Crosbie, J.H.; McDonough, S.M. Optimising engagement for stroke rehabilitation using serious games. Vis. Comput. 2009, 25, 1085-1099. [CrossRef]

20. Cheok, G.; Tan, D.; Low, A.; Hewitt, J. Is Nintendo Wii an Effective Intervention for Individuals with Stroke? A Systematic Review and Meta-Analysis. J. Am. Med. Dir. Assoc. 2015, 16, 923-932. [CrossRef]

21. Corbetta, D.; Imeri, F.; Gatti, R. Rehabilitation that incorporates virtual reality is more effective than standard rehabilitation for improving walking speed, balance and mobility after stroke: A systematic review. J. Physiother. 2015, 61, 117-124. [CrossRef]

22. Lohse, K.R.; Hilderman, C.G.E.; Cheung, K.L.; Tatla, S.; Van der Loos, H.F.M. Virtual Reality Therapy for Adults Post-Stroke: A Systematic Review and Meta-Analysis Exploring Virtual Environments and Commercial Games in Therapy. PLoS ONE 2014, 9, e93318. [CrossRef]

23. Wolfenden, L.; Jones, J.; Williams, C.M.; Finch, M.; Wyse, R.J.; Kingsland, M.; Tzelepis, F.; Wiggers, J.; Williams, A.J.; Seward, K.; et al. Strategies to improve the implementation of healthy eating, physical activity and obesity prevention policies, practices or programmes within childcare services. Cochrane Database Syst. Rev. 2016, 2016, 1-639. [CrossRef]

24. Hutton, B.; Catalá-López, F.; Moher, D. La extensión de la declaración PRISMA para revisiones sistemáticas que incorporan metaanálisis en red: PRISMA-NMA. Med. Clin. 2016, 147, 262-266. [CrossRef]

25. Barcala, L.; Grecco, L.A.C.; Colella, F.; Lucareli, P.R.G.; Salgado, A.S.I.; Oliveira, C.S. Visual Biofeedback Balance Training Using Wii Fit after Stroke: A Randomized Controlled Trial. J. Phys. Ther. Sci. 2013, 25, 1027-1032. [CrossRef] [PubMed]

26. Bower, K.J.; Clark, R.A.; McGinley, J.L.; Martin, C.L.; Miller, K.J. Clinical feasibility of the Nintendo Wii ${ }^{\mathrm{TM}}$ for balance training post-stroke: A phase II randomized controlled trial in an inpatient setting. Clin. Rehabil. 2014, 28, 912-923. [CrossRef] [PubMed]

27. Cho, K.H.; Lee, K.J.; Song, C.H. Virtual-Reality Balance Training with a Video-Game System Improves Dynamic Balance in Chronic Stroke Patients. Tohoku J. Exp. Med. 2012, 228, 69-74. [CrossRef]

28. Hung, J.W.; Chou, C.X.; Chang, H.F.; Wu, W.C.; Hsieh, Y.W.; Chen, P.C.; Yu, M.Y.; Chang, C.C.; Lin, J.R. Cognitive effects of weight-shifting controlled exergames in patients with chronic stroke: A pilot randomized comparison trial. Eur. J. Phys. Rehabil. Med. 2017, 53, 694-702. [PubMed] 
29. Hung, J.W.; Chou, C.X.; Hsieh, Y.W.; Wu, W.C.; Yu, M.Y.; Chen, P.C.; Chang, H.F.; Ding, S.E. Randomized comparison trial of balance training by using exergaming and conventional weight-shift therapy in patients with chronic stroke. Arch. Phys. Med. Rehabil. 2014, 95, 1629-1637. [CrossRef]

30. Kannan, L.; Vora, J.; Bhatt, T.; Hughes, S.L. Cognitive-motor exergaming for reducing fall risk in people with chronic stroke: A randomized controlled trial. NeuroRehabilitation 2019, 44, 493-510. [CrossRef]

31. Karasu, A.U.; Batur, E.B.; Karatas, G.K. Effectiveness of WII-based rehabilitation in stroke: A randomized controlled study. J. Rehabil. Med. 2018, 50, 406-412. [CrossRef]

32. Kim, E.K.; Kang, J.H.; Park, J.S.; Jung, B.H. Clinical Feasibility of Interactive Commercial Nintendo Gaming for Chronic Stroke Rehabilitation. J. Phys. Ther. Sci. 2012, 24, 901-903. [CrossRef]

33. Lee, H.Y.; Kim, Y.L.; Lee, S.M. Effects of virtual reality-based training and task-oriented training on balance performance in stroke patients. J. Phys. Ther. Sci. 2015, 27, 1883-1888. [CrossRef]

34. Lee, H.-C.; Huang, C.-L.; Ho, S.-H.; Sung, W.-H. The Effect of a Virtual Reality Game Intervention on Balance for Patients with Stroke: A Randomized Controlled Trial. Games Health J. 2017, 6, 303-311. [CrossRef]

35. Lee, M.; Shin, D.; Song, C. Canoe game-based virtual reality training to improve trunk postural stability, balance, and upper limb motor function in subacute stroke patients: A randomized controlled pilot study. J. Phys. Ther. Sci. 2016, 28, 2019-2024. [CrossRef] [PubMed]

36. Morone, G.; Tramontano, M.; Iosa, M.; Shofany, J.; Iemma, A.; Musicco, M.; Paolucci, S.; Caltagirone, C. The efficacy of balance training with video game-based therapy in subacute stroke patients: A randomized controlled trial. Biomed Res. Int. 2014, 2014, 580861. [CrossRef] [PubMed]

37. Park, D.S.; Lee, D.G.; Lee, K.; Lee, G.C. Effects of Virtual Reality Training using Xbox Kinect on Motor Function in Stroke Survivors: A Preliminary Study. J. Stroke Cerebrovasc. Dis. 2017, 26, 2313-2319. [CrossRef] [PubMed]

38. Pedreira da Fonseca, E.; Ribeiro da Silva, N.M.; Pinto, E.B. Therapeutic Effect of Virtual Reality on Post-Stroke Patients: Randomized Clinical Trial. J. Stroke Cerebrovasc. Dis. 2016, 26, 94-100. [CrossRef]

39. Rajaratnam, B.S.; Gui KaiEn, J.; Lee JiaLin, K.; SweeSin, K.; Sim FenRu, S.; Enting, L.; Ang YiHsia, E.; KeatHwee, N.; Yunfeng, S.; Woo YingHowe, W.; et al. Does the Inclusion of Virtual Reality Games within Conventional Rehabilitation Enhance Balance Retraining after a Recent Episode of Stroke? Rehabil. Res. Pract. 2013, 2013, 649561. [CrossRef]

40. Singh, D.K.A.; Mohd Nordin, N.A.; Aziz, N.A.A.; Lim, B.K.; Soh, L.C. Effects of substituting a portion of standard physiotherapy time with virtual reality games among community-dwelling stroke survivors. BMC Neurol. 2013, 13, 199. [CrossRef]

41. Song, G.b.; Park, E. cho Effect of virtual reality games on stroke patients' balance, gait, depression, and interpersonal relationships. J. Phys. Ther. Sci. 2015, 27, 2057-2060. [CrossRef]

42. Yatar, G.I.; Yildirim, S.A. Wii Fit balance training or progressive balance training in patients with chronic stroke: A randomised controlled trial. J. Phys. Ther. Sci. 2015, 27, 1145-1151. [CrossRef]

43. Cho, K.H.; Kim, M.K.; Lee, H.J.; Lee, W.H. Virtual Reality Training with Cognitive Load Improves Walking Function in Chronic Stroke Patients. Tohoku J. Exp. Med. 2015, 236, 273-280. [CrossRef]

44. You, S.H.; Jang, S.H.; Kim, Y.H.; Hallett, M.; Ahn, S.H.; Kwon, Y.H.; Kim, J.H.; Lee, M.Y. Virtual reality-induced cortical reorganization and associated locomotor recovery in chronic stroke: An experimenter-blind randomized study. Stroke 2005, 36, 1166-1171. [CrossRef]

45. Wang, C.-Y.; Hwang, W.-J.; Fang, J.-J.; Sheu, C.-F.; Leong, I.-F.; Ma, H.-I. Comparison of Virtual Reality Versus Physical Reality on Movement Characteristics of Persons With Parkinson's Disease: Effects of Moving Targets. Arch. Phys. Med. Rehabil. 2011, 92, 1238-1245. [CrossRef] [PubMed]

46. Baram, Y.; Miller, A. Virtual reality cues for improvement of gait in patients with multiple sclerosis. Neurology 2006, 66, 178-181. [CrossRef]

47. Chang, W.H.; Sohn, M.K.; Lee, J.; Kim, D.Y.; Lee, S.G.; Shin, Y.I.; Oh, G.J.; Lee, Y.S.; Joo, M.C.; Han, E.Y.; et al. Predictors of functional level and quality of life at 6 months after a first-ever stroke: The KOSCO study. J. Neurol. 2016, 263, 1166-1177. [CrossRef]

48. Murie-Fernández, M.; Irimia, P.; Martínez-Vila, E.; John Meyer, M.; Teasell, R. Neurorrehabilitación tras el ictus. Neurologia 2010, 25, 189-196. [CrossRef]

49. De Moya, P.; Bertomeu, B.; Broseta, V. Evaluación y rehabilitación del equilibrio mediante posturografía. Rehabilitacion 2005, 39, 315-323. [CrossRef] 
50. Balaguer García, R.; Pitarch Corresa, S.; Baydal Bertomeu, J.M.; Morales Suárez-Varela, M.M. Posturografía estática con pruebas dinámicas. Utilidad de los parámetros biomecánicos en la valoración del paciente vestibular. Acta Otorrinolaringol. Esp. 2012, 63, 332-338. [CrossRef] [PubMed]

51. Podsiadlo, D.; Richardson, S. The timed "Up \& Go": A test of basic functional mobility for frail elderly persons. J. Am. Geriatr. Soc. 1991, 39, 142-148.

52. Hernández, J.G.; Saldaña, L.M.; Rodríguez, M.P.; Almeida, A.M.; Tejero, J.P.; Alted, C.G. Relación entre las pruebas de funcionalidad de la marcha en personas con daño cerebral adquirido en fase subaguda. AGON Int. J. Sport Sci. 2012, 2, 6-16.

53. Bogle Thorbahn, L.D.; Newton, R.A. Use of the Berg Balance Test to predict falls in elderly persons. Phys. Ther. 1996, 76, 576-583. [CrossRef] [PubMed]

54. Berg, K. Measuring balance in the elderly: Preliminary development of an instrument. Physiother. Can. 1989, 41, 304-311. [CrossRef]

55. Blum, L.; Korner-Bitensky, N. Usefulness of the Berg Balance Scale in Stroke Rehabilitation: A Systematic Review. Phys. Ther. 2008, 88, 559-566. [CrossRef] [PubMed]

56. Wan, B.; Huang, R.; Xiong, W.; Miao, C.; Zhang, F. Meta-analysis of clinical efficacy of postoperative rehabilitation nursing for patients with cerebral hemorrhage. Acta Microsc. 2020, 29, 820-829.

57. Hugues, A.; Di Marco, J.; Ribault, S.; Ardaillon, H.; Janiaud, P.; Xue, Y.; Zhu, J.; Pires, J.; Khademi, H.; Rubio, L.; et al. Limited evidence of physical therapy on balance after stroke: A systematic review and meta-analysis. PLoS ONE 2019, 14, e0221700. [CrossRef] [PubMed]

58. Dee, M.; Lennon, O.; O'Sullivan, C. A systematic review of physical rehabilitation interventions for stroke in low and lower-middle income countries. Disabil. Rehabil. 2020, 42, 473-501. [CrossRef] [PubMed]

59. Kwakkel, G. Intensity of practice after stroke: More is better. Schweiz. Arch. Neurol. Psychiatr. 2009, 160, 295-298.

60. Adamovich, S.V.; Fluet, G.G.; Tunik, E.; Merians, A.S. Sensorimotor training in virtual reality: A review. NeuroRehabilitation 2009, 25, 29-44. [CrossRef]

(C) 2020 by the authors. Licensee MDPI, Basel, Switzerland. This article is an open access article distributed under the terms and conditions of the Creative Commons Attribution (CC BY) license (http://creativecommons.org/licenses/by/4.0/). 